\title{
Assessment of progress of the Postabortion Care Initiative in Francophone Africa
}

\author{
Thierno Dieng \\ Mohamed Diadhiou \\ Nafissatou J. Diop \\ Population Council \\ Youmane Niang Faye \\ Population Council
}

Follow this and additional works at: https://knowledgecommons.popcouncil.org/departments_sbsr-rh

Part of the International Public Health Commons, Maternal and Child Health Commons, Obstetrics and Gynecology Commons, Social and Behavioral Sciences Commons, and the Women's Health Commons

How does access to this work benefit you? Let us know!

\section{Recommended Citation}

Dieng, Thierno, Mohamed Diadhiou, Nafissatou J. Niang Diop, and Youmane Niang Faye. 2008.

"Assessment of progress of the Postabortion Care Initiative in Francophone Africa," FRONTIERS Final Report. Washington, DC: Population Council. 


\title{
Assessment of Progress of the
}

\section{Postabortion Care Initiative in Francophone Africa}

\author{
Centre de Formation et de Recherche en Santé de la Reproduction (Ceforep) \\ Thierno Dieng \\ Mohamed Diadhiou
}

\author{
Frontiers in Reproductive Health (FRONTIERS) \\ Nafissatou J. Diop \\ Youmane Faye
}

\begin{abstract}
April 2008
This study was made possible by the generous support of the American people through the United States Agency for International Development (USAID) under the terms of Cooperative Agreement No. HRN-A-00-98-00012-00 and Subaward No. AI06.058A. The contents are the responsibility of the FRONTIERS Program and do not necessarily reflect the views of USAID or the United States Government.
\end{abstract} FRONTIERS 


\section{EXECUTIVE SUMMARY}

In 2002, a consortium of international and regional partners, comprising CEFOREP (Centre de Formation et de Recherche en Santé de la Reproduction / Center for Training and Research in Reproduction Health), the USAID-funded projects Advance Africa, FRONTIERS, POLICY, PRIME, and SARA, the organizations EngenderHealth, Family Care International , Ipas, JHPIEGO, Population Reference Bureau, the development partners USAID and SIDA, and WHO, established the PAC Initiative for Francophone Africa Committee. The committee seeks to address the programmatic issues of increasing access to and quality of postabortion care (PAC) in Francophone Africa. The first activity of this Initiative was a four-day conference held in Dakar, Senegal in March 2002, which had as its ultimate objective the establishment of quality, sustainable and accessible PAC services throughout the region. The conference report, "Issues in Postabortion Care: Scaling- Up Services in Francophone Africa" summarizes the key issues involved in expanding PAC services in Francophone West Africa ${ }^{1}$.

The Dakar conference provided an opportunity for identifying and reiterating the most appropriate and effective approaches for implementing various aspects of PAC. At a meeting of country "Focal Point" persons for PAC, held in December 2004 in Cotonou, Benin, it became apparent that Senegal, Burkina Faso and Guinea were the countries in the region where the most progress had been made in introducing and rolling out PAC services, while other West African countries were in earlier phases of advocacy or introduction. The meetings in Dakar and Cotonou also revealed that all the countries participating in the Initiative face difficulties in providing PAC, regardless of their stage of implementation.

To address this situation, a meeting was organized in March 2006 in Dakar, attended by CEFOREP, WHO, USAID, AWARE-RH, IntraHealth International, Health Policy Initiative (Futures Group), Africa's Health in 2010, Extending Service Delivery Project, FRONTIERS, MSH and Ipas. The goal was to accelerate implementation of PAC in Francophone African countries to reduce maternal mortality. During this meeting, key strategies and numerous tools for introducing and implementing PAC programs were presented, among them information from the Global Fund, which enriched and enhanced the existing materials.

This study was initiated in response to the needs identified in this meeting by FRONTIERS and was implemented by CEFOREP in six countries: Burkina Faso, Guinea, Mali, Niger, Senegal and Togo. The goals of the study were to:

- Assess the national situation concerning PAC in the six countries ;

- Describe the process of introduction, integration and decentralization of PAC in the six countries;

- Analyze the successes and obstacles in the process of introducing PAC in the countries;

- Recommend key steps that all countries could follow to strengthen the introductory process.

${ }^{1}$ See the introduction of the analytic report of the Dakar Conference: Issues in Post abortion Care: Scaling-Up Services in Francophone Africa, Pape Gaye, IntraHealth International, PAC Consortium, USAID, 2004, pp. vivii. 
The study used two approaches: a comprehensive review of the introduction and institutionalization of PAC in the six countries, and a situation analysis of PAC services within each country. CEFOREP, which hosts the secretariat of the initiative, undertook a documentary review of all relevant reports provided by the six countries; two staff members also visited and conducted in-depth interviews with key stakeholders in each country. Within each country, a case study approach was used to gather information and assess the PAC program in terms of its functioning, coverage and utilization. The assessment process was participatory, eliciting input from all relevant stakeholders to ensure that their perspectives were reflected. The aim was to gauge the changes made and document the lessons learned from the introductory process to inform similar efforts in the future. A total of 48 program/institution managers and 63 service providers were interviewed with the assistance of the country PAC Focal Point person and MOH staff; eight discussion groups were organized with community members.

\section{Results}

The process of introducing PAC in Francophone African countries has followed several approaches. The success of introducing and implementing PAC in these countries was primarily dependent on the good will and efforts of one or two key stakeholders, or "champions", who at times had to overcome numerous challenges because of the sensitivity of the service. In Senegal and Burkina Faso, PAC was originally introduced through pilot Operations Research (OR) projects by the Population Council and JHPIEGO, with support from USAID. These OR projects were conducted in collaboration with the Reproductive Health Department of the Ministry of Health in each country, and with CEFOREP in Senegal and the CRESAR (Cellule de Recherche pour la Santé de la Reproduction) network in Burkina Faso.

To facilitate acceptance of this major innovation, PAC was integrated into the national Emergency Obstetric Care strategy (EmOC) in each country to avoid becoming yet another vertical program without effective links to other related services. The participatory approach of using research to introduce PAC in Senegal and Burkina Faso helped facilitate discussion within the health ministries.

Subsequently, stakeholders in other countries, such as Guinea and Niger, used the results of Senegal and Burkina Faso's experiences to advocate with their own ministries of health. In all four countries, advocacy emphasized the importance of family planning counseling and of making contraceptives immediately available after MVA (manual vacuum aspiration), thus contributing to an increase in the prevalence of postabortion contraceptive use. This was particularly true in Niger, where several broadcast debates and discussions about PAC convinced ministry authorities, parliamentarians, as well as religious leaders, about the benefits PAC could offer to the health system.

In Mali, introduction of the PAC program in 2006 within the $\mathrm{MOH}$ was eclipsed by initiatives by private providers who began using MVA with syringes that were individually purchased and so the $\mathrm{MOH}$ tried to regulate and standardize PAC services.

In all settings, international technical assistance / cooperating agencies have played an important role, through identifying key committed people in influential positions (ministries, parliamentarians, universities, etc.) and supporting sensitization and awareness-raising activities 
for them, as well as supporting and facilitating dialogue between stakeholders and key decision makers.

Partner organizations have supported the introductory activities in accordance with their respective mandates. For example, FRONTIERS has supported operations research that has generated information that contributed to enhancing understanding about the nature the problem and evidence of the feasibility and effectiveness of alternative models, which helped increase interest in adopting new approaches to providing PAC. Organizations such as JHPIEGO (in Burkina Faso, Senegal and Guinea), MSH (in Guinea and Senegal), FCI (in Burkina Faso and Niger), UNFPA (in Burkina Faso, Senegal and Niger), EngenderHealth (Senegal), IntraHealth (Senegal) or the regional USAID-funded AWARE-RH project (in Mali and Togo), have supported training of trainers and of service providers, reorganization of services and supplying programs with MVA equipment and contraceptive products.

In addition, there have been opportunities for organizations to work together in a country to implement the program. For example, this was the case in both Burkina Faso and Senegal, where a coalition comprised of three organizations worked together to help remove political, organizational and technical obstacles. In Burkina Faso, the Yalgado Hospital lacked family planning services and so CRESAR requested support from UNFPA, which provided infection prevention equipment and supplies, financed the construction of, and provided equipment for, a Family Planning Unit, and equipped the surgical unit with equipment and supplies for female sterilization, and from JHPIEGO, which provided MVA equipment.

In both Senegal and Burkina Faso, the gynecologists from the public hospitals were the first to champion PAC in the country. Not only have they been active participants in terms of advocacy, through providing data that so poignantly illustrated the consequences of unsafe abortions, they have also contributed to demonstrating the effectiveness of an integrated approach and to its institutionalization by incorporating PAC into various training curricula.

The support of the partner organizations has been under the supervision of the Ministry of Health in all countries. For example, to facilitate collaboration between the different actors, Technical and Steering Committees were established in Niger and Mali, headed by a MOH representative. The committee members were those generally considered to be key players in the sensitization and mobilization of the authorities and service providers.

Decentralization and/or scaling-up of PAC. The first countries to have introduced PAC (Burkina Faso, Senegal, Guinea and Niger) went through pilot phases to introduce the services that were implemented in national-level institutions. In Mali and Togo, where services were introduced in 2006, the approach has been to include decentralized health facilities from the beginning of the process.

Even though national leaders in RH services have expressed a desire to increase access to PAC services throughout their countries, there were no formal plans from which all the partners could define their strategies and actions. Thus the move towards scaling-up PAC services has not been made at the same speed in all countries. For example, in Senegal scaling up began at the end of a pilot project funded by UNFPA, whereas in Burkina Faso, after the initial introduction of PAC, there was a very long delay before scaling-up started because of insufficient technical assistance and funding. This appears to have been due to a lack of communication among the various 
partners and raises the issue of who should take leadership, which according to one source, has been lacking within the ministries of health. In Senegal, given the very sensitive nature of this service, the supporters in the Division of Reproductive Health decided that the first health facilities to provide PAC would be those with highly respected medical staff and personnel in the maternity units with the strong technical skills.

Two approaches have been taken in the transition to scaling-up PAC services. Some countries have adopted a centralized approach, with a single contact for all stakeholders and partners, while in others the initiative for setting priorities and funding activities has been left to the local authorities. In the centralized approach, which has been the most widely used, it has been the national level of the $\mathrm{MOH}$ that coordinates all the activities but continues to rely heavily on assistance from donors; this approach was followed in Burkina Faso, Niger and Senegal. The decentralized approach has sought to empower the local authorities to develop PAC services, which offers the possibility of more quickly moving on from dependency on outside funding and to more easily involving communities. This approach was envisioned in Mali; to accomplish this, however, would require the creation of a legislative framework that would allow the transfer of resources and expertise.

PAC services are now available in all the regional hospitals in Burkina Faso, Senegal and Guinea. The majority of the district health centers, and many health posts, have also been covered. Between 2003 and 2006 in Senegal, 23 health centers and 300 health posts have benefited from the support of MSH in training staff, reorganizing services, and providing treatment. These facilities were located in the regions supported by USAID: Thies, Louga, Kaolack, Fatick and Ziguinchor. By the end of the program, all health centers and 72 percent of the health posts had been covered.

All the countries had a fairly clear vision of the goals to be achieved over the next four or five years if support is provided by partners. Mali and Guinea intend to engage private health providers in the scaling-up process, which is not the case in Senegal, Niger or Togo.

Degrees of Institutionalization of PAC: All of the countries have documents describing a policy, norms and standards in Emergency Obstetric Care, including PAC. These documents have been, or are in the process of being, revised to include the Five Component Model of PAC: Treatment; Counseling; Contraceptive and Family Planning services; Reproductive and other health services; and Community and Service Provider partnership. The training module for PAC has been standardized by AWARE-RH and adapted and integrated into the in-service training curricula of certain countries.

In Burkina Faso, the emphasis is on counseling, prevention of infections, links with reproductive services and the practice of MVA. In Guinea, it has been integrated into a package of training activities carried out in a chronological order: infection prevention; contraceptive technology; preparation consultation; and then post abortion care. In Senegal training was developed for three types of service providers: Midwives, Head Post Nurses, and Family Planning Counselors. In Senegal and Mali, financial support for training has been made available to the University Public hospitals by Ipas. Integration of the PAC modules into the basic training curricula of health practitioners has not reached the same level in all countries. For example, the midwives in Burkina Faso received training that addresses, among other aspects, prevention of infections and the use of MVA, but this not yet the case in other countries. 
At the university level, this integration has not yet happened in any of the countries, although reforms are in process. This is true for Niger, where the startup is scheduled for the beginning of the academic year 2007-2008. In addition, the West Africa Health Organization (WAHO) is harmonizing these approaches so as to integrate them into the training modules for physicians. However, implementation of this policy may encounter some obstacles owing to the limited capacity of practicum sites in relation to the larger number of trainees.

In the countries where PAC has been available for some time (Burkina Faso, Guinea, and Senegal), PAC services are included in the annual district plans of the MOH. In Burkina Faso and Niger, development partners support staff training and replacement of equipment at lower level facilities. In Mali, Togo and Niger, the process of introducing and scaling- up PAC services is still too recent to include them in district level plans.

Supervision: One of the major problems faced in the process of expanding PAC services is the inability to maintain a system of regular supervision that is appropriate for monitoring provision of PAC services. For example, after the initial follow-up visits after training, a health provider can sometimes go for as long as six months without receiving a visit from a supervisory team. Given that integrated supervisions are becoming increasingly integrated, during which all components of reproductive health are supposed to be reviewed, this often results in supervision sessions that only scratch the surface of problems and the reports from which only offer general recommendations. It should be noted that Guinea does have a regular, parallel system of supervision specific to PAC, but its sustainability remains uncertain as it is dependent on funding from development partners. In Senegal and Togo, regional supervisors appear not to have a clear vision of the PAC model, in contrast to the service providers. This highlights the need to involve them in the training of service providers, so that even if they do not often provide PAC they can perform quality supervision.

Training Policy for PAC: According to those interviewed, no written policy documents exist to guide training in PAC. The health workers targeted to be trained in PAC are generally gynecologists and midwives. However, to increase the number of providers available to provide PAC, other categories are also being trained, such as skilled doctors and nurses in Senegal, nurse-midwives in Mali, and auxiliary health workers and general practitioners in Burkina Faso.

The duality that exists between in-service and pre-service training for reproductive health generally also arises for PAC. In-service training is the main approach used, which is necessary to enhance the knowledge and skills of existing service providers in new therapeutic approaches. Because of this, even if PAC is included in pre-service Emergency Obstetrics Care (EmOC), specific training in PAC is still conducted. In-service training, which is usually organized as seminars and workshops, is costly and often reduces the availability of personnel to offer services for the period of training, particularly in remote areas which already suffer from a lack of staff. Dependence on donor funds for in-service training is a problem, as is the effectiveness and sustainability of this approach to building capacity of human resources.

This is felt particularly at the primary level, where three problems have significant impact on the effectiveness of an in-service training strategy: i) cost of training, which is generally supported by donors; ii) mobility of personnel, which requires repeated training whenever there are new staff; and iii) monitoring of the personnel to assure maintenance of good quality services. To this list can be added the fact that trainees often lack opportunities to practice the new skills given the 
low rate of attendance for PAC in facilities located in remote areas; this has been particularly noticeable in Senegal and Niger. To remedy this problem, practicing using dummy models is considered as an effective alternative training method, but these models are often lacking, as was noted in Niger.

The main challenge for some managers to provide in-service training is creating regional training teams, which could help reduce training costs, cut down the time people have to be absent from their posts to attend training, and assure more regular supervision of the services being offered. In the countries visited, training teams included doctors and midwives, the majority of which were trained at the national level. However, in the areas supported by UNFPA and the USAIDfunded projects, there are training teams that also provide supervision. The training teams available are as follows: Burkina Faso (10), Senegal (19), Guinea (12), Mali (2), Niger (8), and Togo (10). The regional training teams are often subject to high staff turnover rates.

Faced with insufficient resources for organizing PAC training seminars, several facilities have initiated efforts to train their personnel using staff that have previously been trained. This approach, commonly called "cascade training", helps reinforce the capacity of service providers at a lower cost. This approach is used in all the facilities visited and in each the service providers confirmed that cascade training has increased their ability to offer PAC services; in certain facilities, they are now able to offer PAC services around the clock. This approach needs to be documented, and then structured and supported by donors and the supervising institutions. In Senegal in particular, this type of training does not seem to have given the expected results - in several facilities the personnel that have undergone training by their peers have said that they felt the training was truncated.

Health Information System: Data on PAC services are systematically recorded in all the facilities visited. The data collection methods are standardized in Senegal, Burkina Faso and Guinea, while in the three other countries the data are recorded according to the instructions of the trainers. However, in certain facilities, compilation of data is done in the delivery rooms or surgical units where women are treated for abortion complications, which poses a problem especially for recording information on any other services provided. In several locations a codified and institutionalized system does not really exist for collecting the data, thus accurate record keeping relies on the will of a few individuals. It is also important to underscore the weakness of the referral system in all six countries; it is rare that post-treatment feedback is conducted in a systematic and institutionalized manner, and the use of cross-referenced files is practically non-existent.

Supply of MVA kits: The general agreement of those interviewed is that no country has as yet a functioning system for supplying MVA materials. This would require, among other things, introducing norms and protocols for the technical characteristics of the types of MVA syringes that should be used. The syringes used in the facilities are generally those which have been supplied during the training of providers. Moreover, purchase of these materials is mostly funded by development partners, except in Mali where it is the MOH that purchases the syringes and gives them to trained service providers. With this initial stock, certain countries are able to offer PAC services for several years where the program has been implemented; this was the case in Burkina Faso, Senegal and Guinea. Once they are worn out, the facilities have difficulty renewing their equipment. Because none of the countries has been able to find a sustainable solution to this problem the supply of MVA kits is still largely funded by partners. 
Methods taken to ensure an adequate supply of MVA materials vary according to the country and the facilities. For example, in Senegal, MVA supply is centralized, whereas in other countries it is at the discretion of the facility head. In most countries, MVA supplies are not included in the country's national essential supply system, except Guinea. Lower-level health facilities, such as the health centers in Senegal and Burkina Faso, have developed mechanisms for self-financing their supplies of medical commodities generally, including MVA kits. Temporary solutions, such as obtaining supplies from private sources have been adopted, but this can lead to a diversity of supplies, which leads to problems of consistent use and reduces their working life.

To make PAC kits routinely available the ministries in all countries are in the process of coming up with effective solutions. This effort is sometimes in collaboration with partner organizations but not always. In Niger, FCI joined with the $\mathrm{MOH}$ in an aware-raising campaign and in providing advice on how to become sustainable. In Guinea, MVA equipment is now included in the list of essential health supplies because PAC is part of the minimum health care package as an element of Basic Emergency Obstetrics Health Care.

Provision of services: All of the facilities visited provide continuous PAC services from Monday to Friday. In certain facilities, night-shift personnel are also providing the service. In other cases, abortion complications are treated with procedures such as D \& C or flushing by untrained personnel. A lack of availability of MVA equipment during the night-shift tends to encourage use of these other methods and is usually not accompanied by counseling, which sometimes leave the patients in a great deal of pain.

In contrast to after-treatment counseling in family planning, counseling prior to, during, and following the evacuation treatment is not systematic. Facilities in the six countries visited provide a range of contraceptive products at the family planning unit, with the exception of implants and IUDs. It should be noted that these contraceptives are not generally available in the treatment rooms or in recovery rooms, with the exception of Guinea.

Statistics show that the referral system needs to be strengthened and improved. Information about where the patient has been referred and on the patient's status is not always available. Partnership with the community calls for providing care in the patent's immediate environment. However, with the exception of very few organizations (FCI, for example), few partners incorporate this aspect into their programs.

Costs: Service costs range between $\$ 16$ and $\$ 25$ (7,500 FCFA to 10,000 FCFA), which includes the contraceptive method chosen but does not include transportation costs for the client. These costs have been mentioned as financial barriers by both women and men. Some women said they decided to go to traditional midwives who charge between $\$ 2$ to \$ 4 (1,000 FCFA and 2,000 FCFA) for their services and since they are located in the community, other costs such as transportation, are minimized.

Client Satisfaction: The majority of service providers interviewed (in Burkina Faso, Mali, Guinea and Niger) said that their patients are pleased with the service received: the waiting time, pain management, and the interaction with the provider throughout out the entire process. However, some problems in quality of services were noted during group discussions with community members, including inhospitality and negative attitudes of some caregivers, as well 
as service costs. Profiles of clients show that they have a wide range of characteristics and include adults as well as adolescents, and married and unmarried women.

\section{Conclusions}

The regional conference on PAC in 2002 was an important first step for the introduction of PAC in countries of Francophone Africa. One outcome of the conference was a recommended model for the introduction of PAC services. In this model emphasis was placed on identification of national champions, advocacy to gain support for PAC services, the need for an initially vertical program, a pilot phase using an operations research approach, and training of trainers. During this six country assessment, these elements were highlighted as key determinants by national stakeholders. In particular the role of an initial OR study and utilization of the findings and the leadership of university professors as facilitating factors for PAC introduction was seen as being highly influential.

Ten years later the context has changed. PAC services are widely accepted in most countries and so the challenges today are around scaling up, sustainability, supervision and re-supply of MVA equipment. Another important issue that countries are facing is the organization of services to offer FP to all clients and to link them with other relevant RH services. Partnerships with communities do not yet appear to be a crucial element for the ministries and their partners. The following recommendations will be shared with national stakeholders, USAID, CAs and other partners.

\section{Key Recommendations}

Based on the findings summarized in this report and in consideration of some of the essential aspects of PAC services, the following recommendations are made. They concern policymakers, stakeholders, and partners and are grouped according to the major themes addressed in this report:

\section{Institutionalization and scaling-up}

- Responsibility for PAC by the national health ministries must be accompanied by a process of supervising trained health workers and integrating PAC into existing services to avoid making it a vertical program.

- Harmonize the health system with the norms and standards for delivery to enable an institutionalized and coherent decentralization of services.

- Establish a clear legislative/legal framework authorizing a transfer of resources and expertise from the national level towards the community level in terms of financial and administrative management.

- Promote a decentralized approach, leading local authorities to define and formulate their own PAC strategies. 


\section{Procurement}

- Integrate supply of MVA equipment into the National Logistics system to ensure a sustainable supply of MVA kits, which is currently lacking.

- Help health facilities establish and implement supply systems that are based on effective cost recovery mechanisms.

- Conduct advocacy within the hospitals to encourage hospital officials start perceive PAC as an integral component of their services rather than an external program.

\section{Training}

- Evaluate and document cascade training to improve the effectiveness of the strategy.

- Support the introduction of PAC training modules in the curricula of pre-service training institutions where health professionals (doctors, nurses and midwives) and improve the capacity of these training centers.

\section{Monitoring and supervision}

- Train trainers in supervision and monitoring and increase the frequency of regional and district team supervision by a facilitator who is located within the region or district.

\section{Quality and continuity of services}

- Bring together the obstetric and neonatal emergency care units (delivery room and surgery) to make it easier to access MVA equipment, packaging, and material for counseling, contraceptive commodities and data collection materials so that they can be managed within the framework of a normal work shift.

- Develop guides and tools targeted to specific service providers, such as counseling for FP, to strengthen interactions between clients and providers before and during treatment.

- Convince those in charge of health facilities to make contraceptives available within the PAC units. The range of methods should be as wide as possible.

- Reduce barriers to access, especially the client fees - according to one client: "C'est un peu cher, tout le monde n'a pas les moyens" ("It's a bit expensive and not everyone has the means to pay").

\section{Links with other services and programs}

- Establish close links between the PAC services and other programs, such as the malaria in pregnancy program, as malaria is the first leading cause of miscarriage in Francophone Africa. 


\section{Community Involvement}

- Take advantage of existing community-based preventative programs (e.g. malaria) to involve the community in the development of PAC services.

- Involving communities in the development, implementation and monitoring of PAC services plays an important role in getting them to support PAC services.

- Involve existing community-based women's groups to offer information on PAC.

- Increase community information and education sessions to address the lack of knowledge about issues such as family planning, maternal health care and the availability of services. Find ways to get men more involved so that they can increase their knowledge and understanding about the health issues and problems that women face.

- Promote accessible transportation services that are financed by communities to help resolve the problem of emergency evacuations.

\section{Research}

- Conduct operations research to help strengthen services. Depending on the country, determine the feasibility, security, acceptability and costs of the introduction of MVA in the health posts which meet the selection criteria.

- In Senegal, for example, Misoprostol is currently being used without guidelines, training, or complete information. It would be very important to evaluate its eventual use as an option for first line treatment. 


\section{TABLE DES MATIERES}

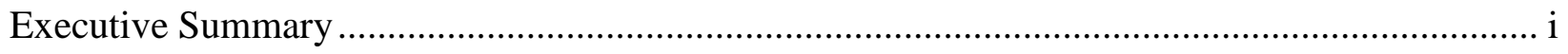

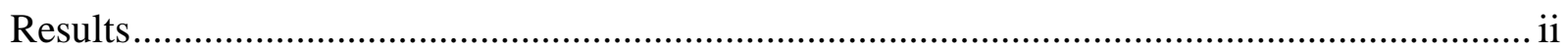

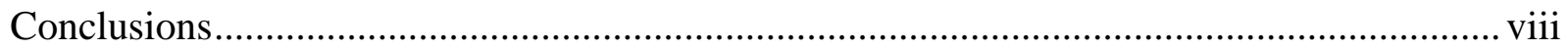

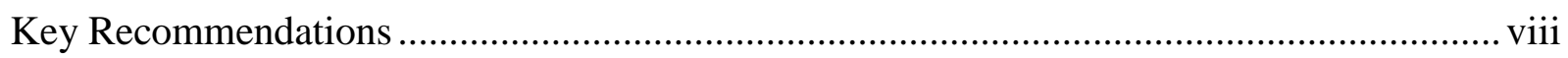

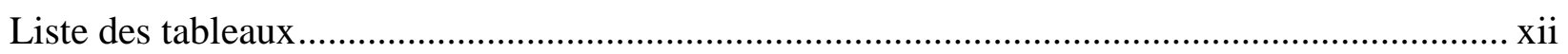

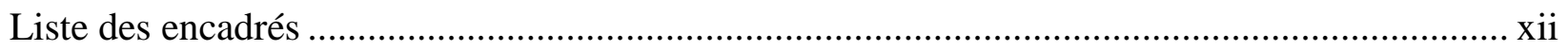

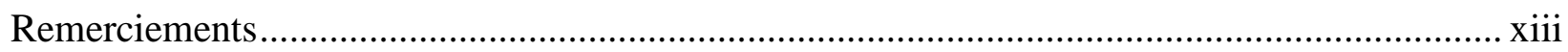

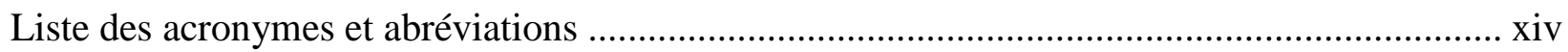

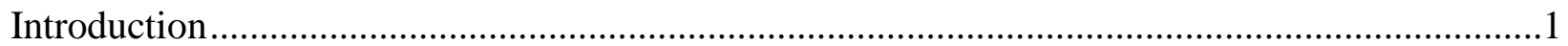

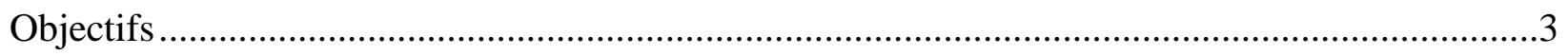

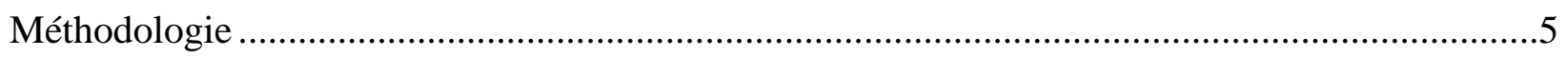

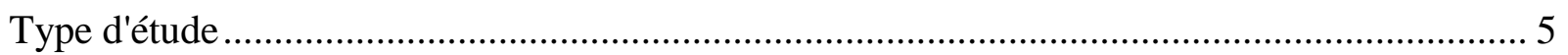

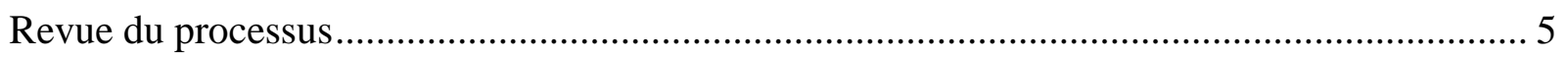

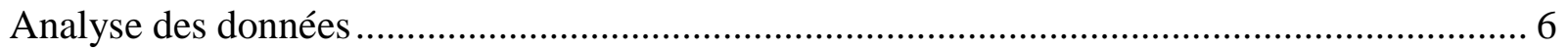

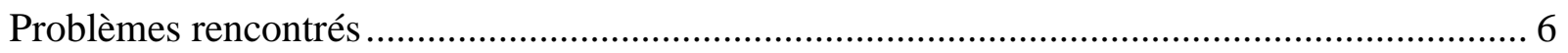

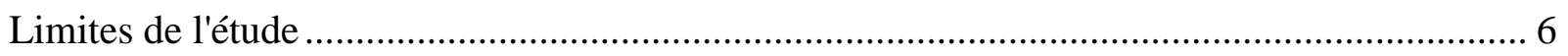

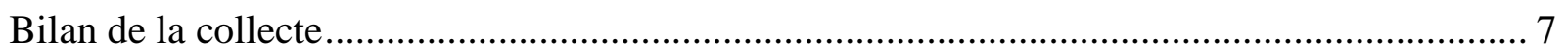

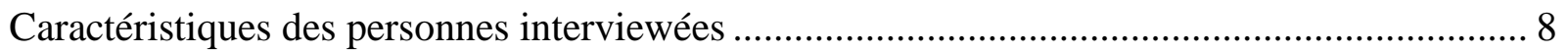

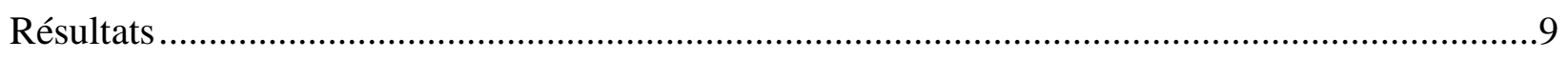

Processus d'introduction des soins après avortement ……............................................... 9

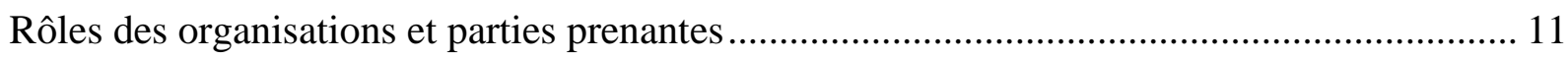

Passage à l'échelle des soins après avortement................................................................. 12

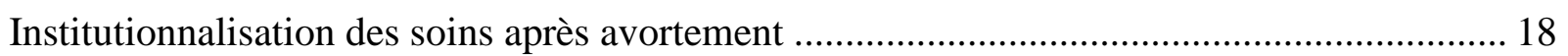

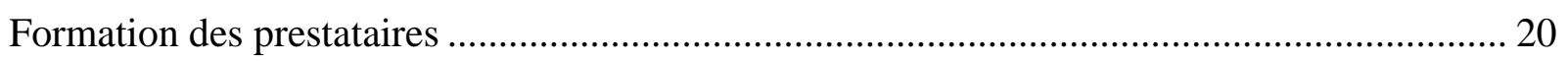

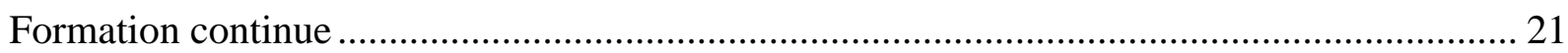

Approvisionnement et équipement en matériels d'Aspiration Manuelle Intra Utérine ............ 25

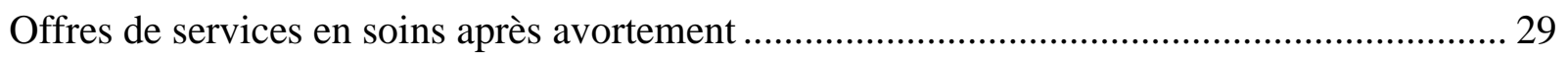

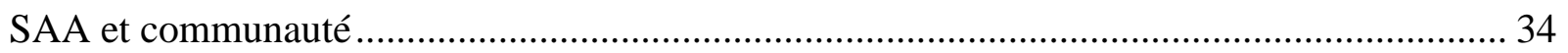

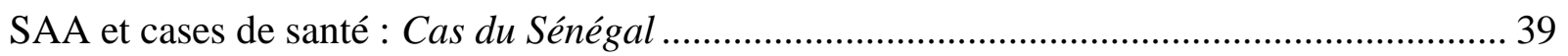

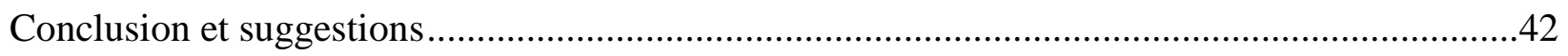

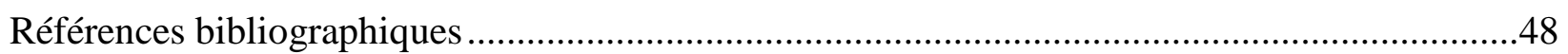

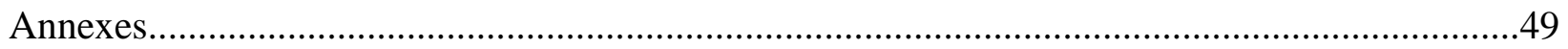

Responsables de programmes et d'institutions interviewés ....................................................... 49

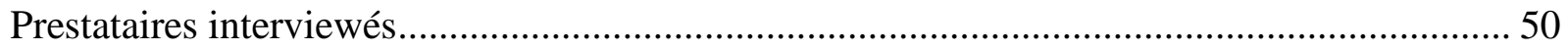




\section{LISTE DES TABLEAUX}

Tableau 1 : Synthèse de la collecte de données ............................................................................ 7

Tableau 2 : Nombre de formateurs en SAA selon les 6 pays de l'évaluation. ..................................22

Tableau 3 : Statistiques sur les activités de SAA dans trois types de structures sanitaires au Sénégal, de janvier à juin 2006.......................................................................... 37

Tableau 4 : Coûts des services de SAA dans les 6 pays selon le niveau des structures sanitaires

\section{LISTE DES ENCADRES}

Encadré 1: Activités de passage à l'échelle des SAA réalisées par MSH au Sénégal ...................17

Encadré 2 : Principales méthodes d'engagement communautaire dans la gestion des complications de grossesse et des soins après avortement 


\section{REMERCIEMENTS}

Nous tenons à adresser nos sincères remerciements à toutes celles et à tous ceux qui, de par leur engagement, ont collaboré à la réalisation de ce travail sur l'évaluation de la situation des soins après avortement en Afrique Francophone. Une réalisation qui aurait été difficile sans le concours et le soutien de divers partenaires.

Nous saluons la franche collaboration des différents partenaires des pays ciblés qui nous ont fourni les informations nécessaires à cette étude. Il s'agit en particulier des responsables des institutions suivantes : les Ministères en charge de la santé au niveau des différents pays (Burkina Faso, Guinée, Mali, Niger, Togo et Sénégal), les Chaires de Gynécologie-Obstétrique des Universités de ces mêmes pays, les agences des Nations Unies, les CAs de l'USAID et les organisations locales affiliées à l'IPPF.

Il faut aussi noter l'assistance et la disponibilité du personnel des structures de santé qui a contribué de façon significative à la réussite de l'étude en facilitant le travail de terrain à l'équipe de recherche.

Nous devons remercier également les enquêteurs et enquêtrices qui ont contribué de façon significative à la réussite de l'enquête au Sénégal, parfois dans des conditions difficiles. Nous associons à ces remerciements, les agents de saisie dont la célérité nous a permis de disposer des fichiers de données dans des temps record. Une motion particulière est à porter au travail de l'opératrice dont la diligence et le professionnalisme ont facilité la transcription des données des interviews et focus des cinq (5) autres pays.

Dans cette étude, nous avons également bénéficié de l'expertise technique du consortium IBP. Sa contribution a été d'un apport certain dans l'élaboration des outils.

Nos vifs remerciements au Groupe de Travail sur les soins après avortement de l'USAID pour son appui financier.

Cette liste n'est pas certainement exhaustive, plusieurs autres personnes ayant apporté leur précise contribution à la réalisation de ce travail. Qu'elles trouvent ici l'expression de notre sincère reconnaissance. 


\title{
LISTE DES ACRONYMES ET ABREVIATIONS
}

\author{
$\mathrm{AC}$ \\ AMIU \\ Agence de collaboration \\ APC \\ ASC \\ AWARE-RH \\ CEFOREP \\ CES \\ CGO \\ $\mathrm{CHU}$ \\ CMA \\ CMC \\ $\mathrm{CO}$ \\ CRESAR \\ CS \\ CSCOM \\ $\mathrm{D} \& \mathrm{C}$ \\ DHN \\ DIU \\ DRS \\ DSF \\ DSR \\ DSVCO \\ EDS \\ ENDSS \\ ENSP \\ ESD \\ FCI \\ IEC \\ INSP \\ IPAS \\ IPPF \\ IST \\ IVA \\ IVG \\ IVL \\ JHPIEGO \\ MAQ \\ $\mathrm{MSH}$ \\ MSPM \\ OMS \\ ONG \\ OOAS \\ PADS \\ PF \\ PI \\ PMA \\ Aspiration Manuelle Intra-Utérine \\ Avortement Provoqué Clandestin \\ Agent de Santé Communautaire \\ Action for West African Region-Reproductive Health (Project) \\ Centre de Formation et de Recherche en Santé de la Reproduction \\ Certificat d'Etudes Spéciales \\ Clinique Gynécologique et Obstétricale \\ Centre Hospitalier Universitaire \\ Centre Médical avec Antenne Chirurgicale \\ Centre Médical Communal \\ Contraceptif Oral \\ Cellule de Recherche en Santé de la Reproduction \\ Centre de Santé \\ Centre de Santé Communautaire \\ Dilatation et Curetage \\ Désinfection à Haut Niveau \\ Dispositif Intra-Utérin \\ Direction Régionale de la Santé \\ Direction de la Santé familiale \\ Direction / Division de la Santé de la Reproduction \\ Direction de la Santé de la Ville de Conakry \\ Enquête Démographique et de Santé \\ Ecole Nationale de Développement Sanitaire et Social \\ Ecole Nationale de Santé Publique \\ Extending Service Delivery Project \\ Family Care International \\ Information Education et Communication \\ Institut National de Santé Publique \\ International Projects Assistance Service \\ International Parenthood Planning Federation \\ Infection Sexuellement Transmise \\ Inspection visuelle à l'acide acétique \\ Interruption Volontaire de grossesse \\ Inspection visuelle au lugol \\ Programme John Hopkins pour l'Education et la Formation Internationale en \\ Gynécologie et Obstétrique \\ Maximiser l'Accès à la Qualité \\ Management Sciences for Health \\ Ministère de la Santé et de la Prévention Médicale \\ Organisation Mondiale de la Santé \\ Organisation Non Gouvernementale \\ Organisation Ouest Africaine pour la Santé \\ Programme d'Appui au Développement Sanitaire \\ Planning Familial \\ Prévention des Infections \\ Paquet Minimum d'Activités \\ PNA \\ Pharmacie Nationale d'Approvisionnement
}




$\begin{array}{ll}\text { PNP } & \text { Politique Normes et Protocoles } \\ \text { PO } & \text { Plan Opérationnel } \\ \text { PPS } & \text { Point de Prestation de Services } \\ \text { PTME } & \text { Prévention de la Transmission Mère-Enfant } \\ \text { REFA PPF } & \\ \text { RO } & \text { Recherche Opérationnelle } \\ \text { RO/AT } & \text { Recherche Opérationnelle/Assistance Technique } \\ \text { SAA } & \text { Soins Après Avortement } \\ \text { SARA } & \text { Soutien à l'Analyse et à la Recherche en Afrique (Projet) } \\ \text { SIDA } & \text { Syndrome d'immunodéficience Acquise } \\ \text { SIG } & \text { Système d'Information et de Gestion } \\ \text { SOU } & \text { Soins Obstétricaux d'Urgence } \\ \text { SOUB } & \text { Soins Obstétricaux d'Urgence de Base } \\ \text { SR } & \text { Santé de la Reproduction } \\ \text { UNFPA } & \text { Fonds des Nations Unies pour les Activités de Population } \\ \text { USAID } & \text { Agence Américaine pour le Développement International } \\ \text { VIH } & \text { Virus d'Immunodéficience Humaine } \\ \text { CA } & \text { Agence de coopération de l'USAID } \\ \text { SanFam } & \text { ONG Santé Familiale }\end{array}$




\section{INTRODUCTION}

En 1997 - 1998, Population Council et Johns Hopkins Program for International Education in Gynecology and Obstetrics (JHPIEGO) ont collaboré avec le Centre de Formation et de Recherche en Santé de la Reproduction (CEFOREP), le Ministère de la Santé au Sénégal et avec la Cellule de Recherche en Santé de la Reproduction (CRESAR) au Burkina Faso pour introduire et tester un modèle pour améliorer les soins après avortement.

Les résultats probants obtenus, notamment au Burkina Faso et au Sénégal, ont poussé un certain nombre de pays à s'intéresser à la question.

Afin d'initier des réponses et des solutions programmatiques aux questions de SAA, un consortium d'agences et de projets internationaux et régionaux, notamment CEFOREP, Advance Africa, FRONTIERS, POLICY, IntraHealth/PRIME, SARA, EngenderHealth, Family Care International, IPAS, JHPIEGO, Population Reference Bureau, l'Agence Suédoise pour le Développement International, USAID, et l'OMS se sont proposés de jouer un rôle catalyseur en créant un comité pour mettre en œuvre une initiative SAA francophone régionale. Le principal but de cette initiative est de promouvoir davantage l'accès et la qualité des services de SAA en Afrique francophone. L'activité centrale de cette initiative a été une conférence majeure de quatre jours à Dakar, Sénégal en mars 2002. Le but ultime de la conférence était d'établir des services de SAA de qualité durables et accessibles dans la région. Le rapport "Questions aux soins après avortement : extension des services en Afrique francophone" résume les questions cruciales liées à l'extension des services de SAA en Afrique francophone de l'Ouest. ${ }^{2}$

Lors de la Conférence de Dakar sur les Soins Après Avortement en 2002, citée ci-dessus, les 14 pays participants avaient élaboré des plans d'action en vue de développer les SAA. La mise en œuvre des plans d'action est appuyée depuis lors par l'Initiative Africaine Francophone pour la promotion des soins après avortement. Cette entreprise a revêtu diverses formes suivant l'état des activités relatives aux SAA. En effet, les pays membres de l'Initiative ne sont pas au même niveau dans la pratique des SAA. La dernière réunion des points focaux qui a eu lieu en décembre 2004 à Cotonou a permis de constater que le Sénégal, le Burkina Faso et la Guinée sont les pays qui ont connu le plus d'avancées, les autres étant globalement dans une phase d'introduction ou bien de prospection. Cependant, les rencontres de Dakar et de Cotonou ont montré que tous les pays de l'initiative francophone sont confrontés à des problèmes, quelle que soit l'étape d'avancement dans laquelle ils se situent.

Le projet de l'USAID dénommé Action for West African Region (AWARE), dont l'un des deux volets est relatif à la santé de la reproduction, a initié un important programme de réplication des expériences réussies dans les 18 pays qu'il couvre. La réplication de la politique de SAA et son renforcement dans sa zone de couverture est l'un de ses axes d'intervention prioritaires.

Dans sa définition et son contenu, le concept de soins après avortement participe à l'amélioration de la qualité des soins par l'implication de tous les acteurs de la santé de la mère. Cette approche

\footnotetext{
${ }^{2}$ Voir l'introduction du rapport analytique de la Conférence de Dakar "Questions aux soins après avortement : extension des services en Afrique francophone", Pape Gaye, IntraHealth International, PAC Consortium, USAID, 2004, page vi-vii.
} 
est novatrice, en ce sens que la mise en œuvre des éléments essentiels des soins après avortement $^{3}$ a entraîné, dans les pays où cela a été expérimenté, d'importantes modifications dans l'organisation des services et dans le comportement des prestataires. On peut citer des aspects importants tels que l'amélioration de l'interaction avec les patientes pour une meilleure gestion de la douleur, un renforcement de l'offre de service de planification familiale après avortement et le respect strict des nouvelles normes de prévention des infections. Des études menées dans différents pays ont prouvé les nombreux gains qu'offre ce paquet de services ${ }^{4}$.

Cependant, le maintien de la qualité des soins et services de SAA à un bon niveau est assujetti à plusieurs conditions telles que la disponibilité du matériel et des produits en quantité et en qualité, et de la disponibilité d'un personnel formé en nombre suffisant. Cette question se pose notamment lors de la décentralisation et aussi dans le cadre de la pérennisation des SAA, comme cela a été noté dans le processus d'extension des SAA au Sénégal ${ }^{5}$ et lors de la Conférence de Dakar. Au-delà de ces conditionnalités, une bonne organisation du travail et une bonne maitrise des différents éléments qui composent le paquet de services sont d'autres facteurs importants pour maintenir la qualité des services.

La mise en exergue de l'importance du rôle de la communauté apporte une dimension supplémentaire à la question de l'implication du bénéficiaire final, pour une plus grande efficacité dans la prise en charge des complications d'avortement. Plus précisément, l'implication de la communauté doit se faire au niveau primaire de la pyramide sanitaire par la détection précoce des signes de fausse couche, mais aussi par l'organisation de la référence vers une structure appropriée $^{6}$. Le rôle des prestataires et de la communauté est tout aussi important s'agissant de la prise en charge des complications d'avortement chez les groupes vulnérables, notamment les adolescentes (femmes de moins de 20 ans) qui représentent $70 \%$ des cas d'avortement dans plusieurs pays d'Afrique ${ }^{7}$. Or, le problème de la gestion de la santé de la reproduction des adolescents en général et des adolescentes en particulier se pose à tous les niveaux de la pyramide sanitaire dans la plupart des pays africains. Par ailleurs, vu l'ampleur de la tâche et les ressources limitées dont disposent les autorités publiques en Afrique, il est nécessaire d'impliquer le secteur privé de la santé dans la planification et la prise de décision concernant les stratégies de prise en charge des complications d'avortement.

A l'évidence, il apparaît que l'introduction et la généralisation des SAA dans les pays africains est un défi quotidien, dans la mesure où, d'une part, les obstacles sont nombreux et, d'autre part,

\footnotetext{
${ }^{3}$ Voir le rapport analytique de la Conférence de Dakar "Questions aux soins après avortement : extension des services en Afrique francophone", PAC Consortium, USAID, 2004, page 7.

${ }^{4}$ Des études pilotes menées en Egypte, au Kenya, au Ghana, au Burkina Faso et au Sénégal ont montré, entre autres résultats, que les SAA permettent de réduire la durée d'hospitalisation, la durée du traitement, les coûts de prise en charge et renforcent la lutte contre les avortements répétitifs par la planification familiale

${ }^{5}$ Voir le rapport de l'étude "Extension des Soins Après Avortement (SAA) : Etude de la référence et de quelques aspects des SAA dans les hôpitaux régionaux de Kaolack, Diourbel et dans le Centre de Santé de Sokone". Ministère de la Santé, Centre de Formation et de Recherche en Santé de la Reproduction (CEFOREP), Fonds des Nations Unies pour la Population (UNFPA) ; $2001 ; 35 \mathrm{p}$.

${ }^{6}$ Une expérience a été menée au Sénégal en 2003-2004 par le Ministère de la Santé et IntraHealth pour tester la faisabilité de la politique de SAA en milieu communautaire.

${ }^{7}$ Voir le rapport analytique de la Conférence de Dakar "Questions aux soins après avortement : extension des services en Afrique francophone", PAC Consortium, USAID, 2004, page 64.
} 
le maintien des acquis n'est pas garanti si les orientations idoines ne sont pas prises. C'est pour contribuer à ce challenge que le Population Council, et le CEFOREP se proposent de mener une étude dans six pays membres de l'Initiative, consistant à faire un tour d'horizon des obstacles institutionnels et techniques à régler afin de réussir la généralisation des soins après avortement en Afrique francophone.

\section{OBJECTIFS}

La présente étude vise les objectifs suivants :

- Evaluer la situation dans six pays de l'initiative

- Décrire le processus d'introduction, d'intégration et de décentralisation des SAA

- Analyser les réussites et les obstacles durant le processus d'introduction, d'intégration et d'institutionnalisation du modèle de SAA

- Recommander les étapes clés que les pays pourraient emprunter pour améliorer la situation.

Plus précisément les questions suivantes seront explorées.

1. Introduction des SAA dans le pays

- Est-ce qu'il y a eu une RO avant l'introduction des SAA ?

- Une approche pilote a t'elle été menée ?

- Quel a été le rôle des institutions, en particulier celui du Ministère de la Santé ?

- Quels sont les "Champions" et /ou leaders qui ont impulsé les SAA ?

2. Niveau d'Institutionnalisation des SAA

- Les SAA sont-ils intégrés dans les documents de Politiques, Normes et Protocoles (PNP)?

- Les PNP sont ils disponibles dans les structures de santé ?

- Les SAA sont ils intégrés dans les curricula de formation de base ?

- Les SAA sont ils intégrés dans les curricula de formation continue?

- Les SAA figurent-ils dans les PO ?

- Quel est le système de financement des SAA dans les pays ?

- Existe-t-il un système d'information qui intègre les SAA ?

- Comment la supervision est-elle menée ? Et par qui ?

3. Passage à l'échelle

- Existe-t-il une vision ou un plan de passage à l'échelle concernant les services de SAA dans les pays visités?

- Quels sont les niveaux atteints concernant le passage à l'échelle selon les pays (Hôpitaux nationaux, régionaux, districts, le secteur privé s'il est impliqué) ?

- Quel a été le niveau de financement du passage à l'échelle selon les pays ?

- Quelles sont les zones couvertes ou non couvertes par les partenaires au développement dans ces pays ?

- Y a t'il eu une décentralisation des SAA ? Dans quelle mesure les transferts de compétences et de responsabilités ont pu être réalisés dans ces pays ? 


\section{Formation}

- Les outils de formation continue sont ils disponibles? Quel est le type de formation utilisé par le MS dans ces pays ?

- La formation comporte-t-elle à la fois un volet quantitatif et qualitatif ?

- Quels sont les niveaux de connaissances et de compétences des formateurs?

- Comment les prestataires apprécient ils la qualité de la formation reçue ?

- Quel est le système de supervision formative dans ces pays ?

- Les prestataires formés sont ils disponibles à tout moment ?

5. Equipement et Approvisionnement

- Quel est le système d'approvisionnement en kits AMIU en vigueur ?

- Ce système est il efficace et pérenne ?

- Quels sont les équipements disponibles en terme de kits AMIU, stérilisateurs, tables, spéculums...?

- Les médicaments (analgésiques, produits contraceptifs) et consommables (gants, matériel de prévention de l'infection) sont ils disponibles au niveau de ces services ?

6. Offre de services de SAA

- Y-a-t'il une continuité dans l'offre de services de SAA ?

- Quel est le modèle de SAA mis en place dans ces pays ?

- L'organisation des services correspond-elle au modèle en place ?

- Quel est le lien entre le service de traitement d'urgence et l'unité PF ?

- Existe-t-il un système de référence et de contre référence entre les structures ?

- Quels sont les prestataires qui interviennent dans l'offre des services SAA dans ces pays ?

- Quels sont les coûts des services SAA ?

- Comment sont organisés le système de collecte des données au niveau des structures de santé et la remontée de l'information?

- Quelles sont les opportunités qui permettent aux prestataires de donner leurs opinions sur les services de SAA ?

- Comment les clientes apprécient-elles les services reçus ?

7. SAA et communauté

- La communauté est-elle informée de l'existence des services de SAA ?

- Comment la communauté est-elle impliquée dans la mise en place des activités SAA ?

- Existe-t-il un système organisé pour les évacuations en cas d'urgence au sein de la communauté ?

- Les SAA sont ils offerts au niveau des cases de santé ?

- Dans quelle mesure la communauté peut elle donner ses opinions et suggestions par rapport à l'accès et à la qualité des services de SAA ?

8. PAC et communautés

- La communauté est elle informée de l'existence des services de SAA ?

- Comment la communauté est-elle impliquée dans la mise en place des activités SAA ? Quel est le degré d'implication des hommes?

- Existe-t-il un système de référence et de contre référence efficace/ fonctionnel au sein de la communauté ?

- Les SAA sont ils offerts au niveau des cases de santé ?

- Dans quelle mesure la communauté peut elle donner ses opinions et suggestions par rapport à l'accès et à la qualité des services de SAA ? 


\section{METHODOLOGIE}

\section{Type d'étude}

Il s'agit d'une étude multicentrique, qui a utilisé deux approches pour faire le point sur la pratique des SAA au Burkina Faso, en Guinée, au Mali, au Niger et au Togo, depuis leur introduction dans ces pays respectifs. La première approche a consisté en une revue complète du processus d'introduction, de généralisation et d'institutionnalisation des SAA dans le système de santé, de même que les mécanismes qui sont utilisés pour, d'une part, assurer l'approvisionnement en matériels de traitement et, d'autre part, les moyens et méthodes utilisés pour assurer le maintien à un bon niveau de la qualité des prestations. La seconde approche a consisté en une analyse situationnelle qui avait pour but de faire le point sur la pratique actuelle des SAA aux différents niveaux de la pyramide sanitaire. Elle a été complétée, dans la mesure du possible, par une petite enquête auprès des clientes, afin de recueillir des informations sur la gestion de la douleur, le counseling en PF et l'offre de produits contraceptifs.

\section{Revue du processus}

Pour mener à bien cette partie, l'équipe de recherche s'est proposée, dans un premier temps, de mener une revue documentaire et, dans un second temps, de mener des interviews auprès de personnes clés qui auront joué un rôle important dans le processus.

\section{Revue documentaire}

Il s'est agi d'une revue des textes officiels (textes de lois, documents de politiques, normes et protocoles), des rapports de missions de supervision et des documents de recherche. Ces données ont permis de comprendre l'environnement juridique et législatif, de mesurer le degré d'intégration des SAA dans les politiques de soins et aussi le volume d'activités en rapport avec les SAA. Les données ont été collectées au niveau de la structure nationale chargée de la politique de SAA, au niveau des structures techniques chargées de la formation des prestataires et de la supervision des activités de SAA, au niveau des institutions d'appui au ministère de la santé et aussi au niveau des structures qui ont mené des activités de recherche en SAA.

\section{Etude de cas}

Dans un second temps, une étude de cas a été menée au Burkina Faso, en Guinée, au Mali, au Sénégal, au Niger et au Togo. Elle avait pour objectifs de faire le point, de façon plus approfondie, sur les aspects institutionnels liés à la décentralisation, l'institutionnalisation, la gestion, la supervision, le plateau technique, la continuité des services, la qualité de la pratique aux plans clinique et para clinique, le niveau de satisfaction des clientes et les mécanismes d'approvisionnement en kits AMIU et en produits liés aux SAA.

\section{Analyse situationnelle}

L'analyse situationnelle avait pour objet d'évaluer le plateau technique des structures ciblées par l'étude. Le respect des normes et protocoles, de même que la qualité des services ont été mesurés par le biais d'un guide d'observation.

\section{Enquête qualitative}

Une enquête qualitative a été menée auprès de personnes clés, par le biais d'interviews approfondies, en vue de collecter des informations sur les actions concrètes réalisées, les 
difficultés rencontrées, ainsi que les mécanismes et ressources utilisés pour les résoudre. Les personnes clés étaient les suivantes :

- Le responsable de la structure chargée, au niveau ministériel, de la question des SAA et son personnel;

- Les responsables des zones sanitaires aux différents niveaux de la pyramide sanitaire ;

- Les responsables de programmes comportant les SAA ;

- Les membres du personnel clinique et paraclinique intervenant dans les SAA ;

- Les membres des communautés représentatives des populations.

\section{Analyse des données}

Compte tenu des différentes approches proposées pour étudier la situation des SAA dans les pays, l'équipe de recherche a procédé à l'analyse des données selon 2 approches :

- Les données issues de l'enquête auprès des personnes clés et des femmes ont été traitées selon la méthode d'analyse des contenus ;

- Les données issues de l'enquête situationnelle ont été analysées selon le procédé classique utilisé par JHPIEGO.

Dans un souci de rendre l'analyse plus en cohérence avec les schémas proposés lors de la Conférence de Dakar, les commentaires seront faits en tenant compte du plan ci-après :

- Les aspects politiques et institutionnels ;

- La mise à l'échelle, y compris la décentralisation ;

- La pérennisation / l'institutionnalisation.

\section{Problèmes rencontrés}

Pour la collecte des données, il avait été prévu de consacrer cinq jours ouvrables dans chaque pays, sauf pour le Sénégal où l'USAID avait souhaité procéder à une étude de plus grande envergure, avec une équipe de collecte plus étoffée. Cela, associé au nombre assez important de responsables et prestataires à interviewer, a fait que les chercheurs n'avaient que peu de possibilités de rechange en cas d'indisponibilité des personnes ciblées.

Cette non disponibilité de quelques personnes ciblées a été une autre des difficultés auxquelles les chercheurs ont quelques fois été confrontés. Dans certains cas il a été possible de trouver des remplaçants.

Par ailleurs, il n'a pas toujours été possible d'avoir des données complètes sur les activités SAA des structures visitées. Seules étaient généralement disponibles les données globales décrivant le volume des activités SAA durant une période assez récente

\section{Limites de l'étude}

Les données de cette évaluation ont été collectées dans 6 pays choisis de manière raisonnée. Donc, même si ces pays représentent, à notre avis, toutes les situations qui se sont produites, elles ne devraient pas être systématiquement généralisables à tous les autres pays de la sous région. 
D'autre part, la collecte d'information a été limitée aux villes capitales hormis au Sénégal. Cela n'a pas permis d'avoir suffisamment d'informations sur le vécu de la pratique des SAA par les acteurs en milieu décentralisé.

L'organisation de focus groups avec les clientes n'a pas été possible dans tous les pays. Seul le Sénégal avait dès le départ bénéficié d'un budget pour ce volet.

\section{Bilan de la collecte}

\section{Tableau 1 : Synthèse de la collecte de données}

\begin{tabular}{|c|c|c|c|c|}
\hline \multirow{2}{*}{ Pays } & \multicolumn{2}{|l|}{ Cibles } & \multirow{2}{*}{ FDG } & \multirow{2}{*}{$\begin{array}{l}\text { Structures sanitaires } \\
\text { visitées }\end{array}$} \\
\hline & Responsables de programme et d'Institutions & Prestataires & & \\
\hline $\begin{array}{l}\text { Burkina- } \\
\text { Faso }\end{array}$ & $\begin{array}{l}\text { - Directeur des Etudes de l'Institut National de } \\
\text { - Manté Publique } \\
\text { - Médecin Chef Maternité CMA } \\
\text { - Conseillère de I'OMS } \\
\text { - Directeur : chef DSF } \\
\text { - Coordonnatrice de FCI } \\
\text { - Chargé de projet FCI dans un District } \\
\text { - Médecin adjoint de la maternité du CHU et point } \\
\text { focal SAA } \\
\text { - Ancienne Présidente du CRESAR } \\
\text { - Chargée de programme UNFPA }\end{array}$ & $\begin{array}{l}\text { - } 2 \text { Médecins }(\mathrm{CHU})+ \\
2 \text { Sages-femmes } \\
\text { - } 2 \text { Médecins }(\mathrm{CMA})+ \\
2 \text { Sages-femmes }\end{array}$ & 2 & $\begin{array}{l}1 \mathrm{CHU} \\
1 \text { Centre de santé }\end{array}$ \\
\hline Mali & $\begin{array}{l}\text { - Directeur : chef DSR } \\
\text { - Point focal SAA } \\
\text { - Médecin chef Maternité CHU } \\
\text { - Médecin chef Centre de référence }\end{array}$ & $\begin{array}{l}\text { - } 2 \text { Médecins }(\mathrm{CHU})+ \\
2 \text { Sages-femmes } \\
\text { - } 2 \text { Médecins (Centre de } \\
\text { référence) + } \\
2 \text { Sages-femmes }\end{array}$ & 0 & $\begin{array}{l}1 \mathrm{CHU} \\
1 \text { Centre de santé }\end{array}$ \\
\hline Guinée & $\begin{array}{l}\text { - Responsable Formation MS Guinée } \\
\text { - Chargé de Programme EngenderHealth } \\
\text { - Chef section à la DSR } \\
\text { - Chef CMC de Conakry } \\
\text { - Enseignant Faculté de Médecine, Point Focal } \\
\text { SAA en Guinée }\end{array}$ & $\begin{array}{l}\text { - } 3 \text { Médecins }(\mathrm{CHU})+ \\
2 \text { Sages-femmes } \\
\text { - } 2 \text { Médecins }(\mathrm{CS})+ \\
2 \text { Sages-femmes }\end{array}$ & 1 & $\begin{array}{l}1 \mathrm{CHU} \\
1 \text { Centre de santé }\end{array}$ \\
\hline Togo & $\begin{array}{l}\text { - Médecin chef Maternité CHU } \\
\text { - Directeur : Chef DSF } \\
\text { - Responsable de programme Association } \\
\text { Togolaise pour le Bien Etre Familial }\end{array}$ & $\begin{array}{l}\text { - } 2 \text { Médecins }(\mathrm{CHU})+ \\
2 \text { Sages-femmes } \\
\text { - } 2 \text { Assistants médicaux }\end{array}$ & 0 & $\begin{array}{l}1 \text { CHU } \\
1 \text { Hôpital urbain }\end{array}$ \\
\hline Niger & $\begin{array}{l}\text { - Administrateur FCl } \\
\text { - Médecin chef Maternité }\end{array}$ & $\begin{array}{l}\text { - } 1 \text { Médecin Adjoint } \\
\text { (Maternité) + } \\
2 \text { Sages-femmes } \\
\text { - } 2 \text { Sages-femmes } \\
\text { (centres de santé) }\end{array}$ & 0 & $\begin{array}{l}1 \mathrm{CHU} \\
1 \text { Centre de santé }\end{array}$ \\
\hline Sénégal & $\begin{array}{l}\text { - } 25 \text { Parties prenantes et Partenaires : IntraHealth, } \\
\text { OMS, UNFPA, MSH (2), Engender Health, } \\
\text { ENDSS, CGO, CEFOREP, Centre de santé Roi } \\
\text { Baudouin, DSR, USAID (2), PNA, Région } \\
\text { médicale Ziguinchor (3), Région médicale Thiès } \\
\text { (2), Région médicale Kaolack (2), District de } \\
\text { Sokone (2), Présidents de Comité de Santé (2) }\end{array}$ & $\begin{array}{l}\text { - } 27 \text { Prestataires : } \\
\text { Sages-femmes et } \\
\text { Conseillères }\end{array}$ & 5 & $\begin{array}{l}2 \text { Hôpitaux régionaux } \\
7 \text { Centres de santé } \\
2 \text { Cases de santé }\end{array}$ \\
\hline TOTAL & 48 & 63 & 8 & 21 \\
\hline
\end{tabular}




\section{Caractéristiques des personnes interviewées}

Dans le cadre de l'évaluation de la pratique des soins après avortement plusieurs personnes, responsables de services cliniques ou de programmes comprenant les soins après avortement ont été interviewées. Les responsables de services cliniques étaient en poste aussi bien dans les structures hospitalières de référence, que dans d'autres ayant le niveau de centre de santé dotés d'une unité chirurgicale ou non. Pour les responsables de programme, il s'agissait des directeurs de service ministériels chargés de la mise en œuvre des SAA ou leurs représentants et des chargés de programmes, dans des ONG ou des organismes du système des Nations Unies, dont les SAA sont une des composantes. Cependant, au Burkina Faso, nous avons rencontré des personnes ressources qui avaient joué un rôle clé dans l'introduction des SAA, mais qui ne sont plus en service.

Dans ce même exercice, l'équipe de recherche a interviewé plusieurs prestataires de services. Il s'agissait de médecins gynécologues et de sages-femmes, tous impliqués dans la prise en charge des femmes présentant des complications d'avortement. Ces prestataires étaient en services aussi bien dans les structures hospitalières de référence, que dans d'autres ayant le niveau de centre de santé doté d'une unité chirurgicale ou non. Généralement, dans chacune de ces structures, deux médecins et deux sages-femmes ont été interviewés.

Le niveau de responsabilité des personnes interrogées variait selon le type de structure. Au niveau du CHU, les médecins interrogés assuraient le rôle de prestataires impliqués dans la prise en charge des soins après avortement, mais également de formateurs, de superviseurs et de responsables dans ce domaine. Quant aux sages-femmes elles étaient respectivement responsables des unités de SAA et prestataires affectées dans lesdites unités de soins après avortement. Pour les centres de santé également, les prestataires interrogés étaient tous responsabilisés dans les domaines cliniques et administratifs. Cependant, nous avons choisi de les interroger selon leur activité dominante dans leur structure et pour les SAA. 


\section{RESULTATS}

\section{Processus d'introduction des soins après avortement}

L'introduction des Soins Après Avortement (SAA) en Afrique francophone a suivi plusieurs approches. Si les premiers pays ont dû réaliser des recherches opérationnelles, les suivants se sont, soit directement inspirés des expériences déjà menées. Le secteur privé a quelque fois précédé l'administration sanitaire qui a plus tard apporté les éléments de stratégie et de normalisation.

\section{Les différentes approches d'introduction des SAA}

Avec la Recherche Opérationnelle : Au Sénégal et au Burkina Faso, les SAA ont été introduits sous forme de recherche opérationnelle (RO) par le Projet de Recherche Opérationnelle et d'Assistance Technique en Afrique II (OR/TA II) en collaboration avec John Hopkins Program for International Education in Gynecology and Obstetrics (JHPIEGO). Ces RO ont été menées avec les Ministères de la Santé des deux pays et le Centre de Formation et de Recherche en Santé de la Reproduction (CEFOREP) au Sénégal et la Cellule de Recherche en Santé de la Reproduction (CRESAR) au Burkina.

L'acceptabilité pour ces RO a été d'autant plus grande que la question des avortements clandestins et ses conséquences préoccupaient les responsables sanitaires de ces pays. Les statistiques d'hôpitaux relevaient des causes de décès maternels dues aux avortements assez élevés $(18 \%$ à $20 \%)$. "...donc il était évident qu'on puisse adresser ce problème pour voir dans quelle mesure on pouvait assurer des soins de qualité aux femmes qui sont victimes de complications d'avortement avant de penser à leur offrir une méthode contraceptive qui puisse les aider à éviter une grossesse non désirée." (Chargé de programme, Burkina Faso)

Cependant, le contexte juridique et socioculturel rendait très sensible les questions relatives aux avortements. En effet, sous l'angle juridique, les lois relatives au sujet interdisaient de manière stricte le recours à l'interruption de grossesse. La révision de la loi française de 1920 et l'adoption du Code sur la santé publique au Burkina ont permis de considérer la planification familiale comme une question de santé publique mais interdisaient toujours tout recours à l'avortement. A côté de cet aspect juridique, la forte prégnance des leaders religieux sur les attitudes et croyances des populations, représentait un autre obstacle de taille pour instaurer un débat fructueux sur la question.

La démarche participative adoptée par les précurseurs de la recherche au Sénégal et au Burkina a facilité l'élargissement des discussions au sein du Ministère de la Santé et de certains responsables de programme. "Même si les textes et lois interdisaient formellement tout recours à l'interruption volontaire de grossesse (sauf si le pronostic vital maternel était engagé), ces derniers proclamaient la nécessité de prévenir la mortalité maternelle" (Responsable de programme, Burkina Faso). Cette brèche a été utilisée par les promoteurs des SAA qui ont mis en exergue l'importance du volet counseling et des dispositions prises pour offrir une méthode contraceptive dans l'immédiat.

Les résultats des RO dans 2 hôpitaux et 1 centre de santé au Sénégal et 2 hôpitaux au Burkina ont fini de convaincre les sceptiques : la faisabilité d'introduire les SAA, la réduction des coûts d'hospitalisation et du coût pour le client, l'augmentation de l'utilisation de la contraception ont 
fourni des arguments solides. Les recherches ont aussi révélé la qualité des services offerts aux patientes souffrant de complications d'avortement, particulièrement celles soupçonnées d'IVG. Ce sont ces cas difficiles qui interpellent, encore à ce jour, l'éthique médicale.

Utilisation de l'expérience des autres. En 1998, après la conférence de Maurice où les résultats des RO furent partagés, la délégation guinéenne composée de techniciens a cherché le soutien du Ministère de la Santé pour entamer le processus d'introduction. L'appui de JHPIEGO a été un plus dans cette démarche.

Le plaidoyer auprès des autorités locales politiques, administratives, coutumières et religieuses a consisté à montrer les dérives possibles de la méthode de dilatation et curetage, qui a été responsable d'une morbidité et d'une mortalité importantes en Guinée. Si des dérives d'utilisation advenaient avec l'AMIU, les risques de complications seraient moindres. En plus l'offre systématique de la planification familiale a suscité de l'intérêt. "C'est que déjà au Ministère de la Santé, ils étaient très sensibilisés sur l'effet de la planification familiale, et tout le monde demandait comment faire pour augmenter la prévalence contraceptive. Donc cela a été immédiatement vu comme une voie pour augmenter la prévalence contraceptive." (Universitaire, Guinée)

En Guinée, c'est donc suite à la capitalisation des résultats des RO du Burkina et Sénégal, que les SAA ont été introduits dans 2 hôpitaux. Les premières formations ont été faites dans ces 2 pays. " Ce n'était pas vraiment une étude opérationnelle, comme la $R O$, mais c'était pour voir comment sur le plan logistique, sur le plan organisationnel, comment on pourrait mettre en place une phase test" (Universitaire, Guinée).

Initiatives privées puis standardisation par le Ministère. Au Mali, l'introduction des SAA a été favorisée par un engagement politique, au plus au niveau de l'Etat, dans les grandes initiatives de réduction de la mortalité maternelle telles que la Vision 2010. Les hautes autorités ministérielles, suite à des rencontres régionales durant lesquelles la question a été abordée, ont aussi interpellé la Direction de la Santé de la Reproduction sur la nécessité d'introduire cette nouvelle approche. Cette dernière avait déjà entamé des démarches à partir de 2003 auprès du projet AWARE-RH et de la mission locale de l'USAID pour la mise en place de ce programme.

Selon plusieurs répondants, une bonne partie des gynécologues utilisait déjà la seringue AMIU qu'ils s'étaient procurés individuellement lors des conférences régionales. Ainsi, le Ministère devait surtout procéder à une standardisation de la prise en charge des complications d'avortement, avec la formation de plus de personnel sur l'ensemble du paquet d'activités, la réorganisation des services afin d'améliorer l'accès et la qualité des soins, et de rendre plus rigoureuse la gestion du matériel et du système d'information (notification systématique des cas). Avec le financement du projet AWARE-RH et l'assistance technique de CEFOREP, ce travail a pu être fait en 2006.

Initiative pionnière. L'idée d'introduire les SAA au Niger remonte à la Conférence Maximiser l'Accès à la Qualité (MAQ) de Dakar (1998), durant laquelle une équipe du Ghana avait fait une présentation sur les SAA. Par la suite, une conférence a eu lieu à Tawa au Niger (1999) avec la DSR, FCI et UNFPA sur les Soins Obstétricaux d'Urgence (SOU). Au cours de cette conférence, il y a eu beaucoup d'interrogations sur le lien potentiel entre SAA et IVG. L'idée d'introduire les SAA au Niger avait été exprimée par le médecin chef de la maternité I. Gazobi lors de cette 
rencontre. Cette volonté s'est heurtée à de nombreuses réticences et pressions de la part de certains collègues médecins. Cependant, de nombreux échanges doublés de débats radiotélévisés et de démonstrations sur la méthode ont convaincu les autorités ministérielles, les parlementaires de même que les leaders religieux sur les bénéfices que pourrait procurer les SAA au système de santé. Son introduction a été faite par le développement de PNP en 2002.

\section{Rôles des organisations et parties prenantes}

\section{Les Agences de collaboration (CAs) et UNFPA}

L'introduction des SAA dans les pays a été initiée par les CAs en s'appuyant, toutefois, sur l'identification de personnes clés occupant des fonctions au Ministère de la Santé ou au sein de l'université. Parfois il s'agissait de parlementaires. Un travail d'explication du concept de soins après avortement a été entrepris auprès de ces personnes, en vue de les sensibiliser sur l'importance de la question et sur l'utilité d'agir. Avec l'aide de ce premier noyau restreint ainsi créé, un plaidoyer était entrepris envers les décideurs.

Les CAs ont appuyé financièrement et techniquement les actions de plaidoyer par la facilitation du dialogue entre les différents acteurs et décideurs, par l'organisation de recherches opérationnelles, de rencontres d'information et de sensibilisation. A ce propos, leur expertise dans le processus d'utilisation des résultats de recherche a été déterminante. Une fois l'idée d'introduire les SAA acceptée par les décideurs, les CAs et UNFPA ont poursuivi leurs actions en apportant un appui technique, matériel et financier pour la planification du processus, la mise en place des activités du programme, et aussi l'évaluation des activités.

Dans tous les pays visités, ces organismes continuent à jouer un rôle primordial dans la continuité des activités de SAA. Ceci, grâce à l'appui qu'ils apportent aux structures dans la formation des prestataires, dans l'équipement en matériel AMIU, mais aussi dans l'institutionnalisation et la standardisation des SAA dans les Politiques, Normes et Protocoles.

Il faut néanmoins préciser que les organismes partenaires intervenaient en fonction de leur mandat. Ainsi, Population Council a contribué, par la recherche opérationnelle, à fournir des informations importantes qui ont permis de comprendre l'ampleur du problème, et l'intérêt d'adopter la nouvelle approche de prise en charge des complications d'avortement. Des institutions comme JHPIEGO (Burkina Faso, Sénégal, Guinée), MSH (Guinée, Sénégal), FCI (Burkina Faso et Niger), l'UNFPA (Burkina Faso, Sénégal, Niger), Engender Health (Sénégal), IntrahHealth (Sénégal) ou le projet régional AWARE-RH (Mali, Togo) ont contribué à la formation des formateurs et des prestataires, à la réorganisation des services, de même que l'approvisionnement des structures en matériels AMIU et en produits contraceptifs. Ces actions étaient limitées aux zones d'intervention de chaque organisme dans un pays. De plus, il est arrivé que plusieurs institutions travaillent ensemble dans un pays pour mettre en place le programme. Ce fut le cas au Burkina Faso et au Sénégal où la coalition entre trois institutions a permis, dans chacun de ces deux pays, de lever plusieurs obstacles politiques, organisationnels et techniques. Au Burkina Faso, puisque la maternité du CHU Yalgado ne disposait pas d'unité de planification familiale, la CRESAR a fait appel à l'UNFPA. Cette institution a fourni les produits pour la prévention des infections, a financé la construction et l'équipement de l'unité de PF et a équipé le bloc opératoire de la maternité en matériel pour la pratique de la contraception chirurgicale volontaire. JHPIEGO a fourni le matériel AMIU. 


\section{Le rôle des professeurs d'Université}

Dans des pays comme le Sénégal et le Burkina, ce sont les professeurs gynécologues des CHU qui ont été le premiers "champions des SAA". Ils ont non seulement participé au plaidoyer en fournissant des chiffres éloquents des conséquences des avortements mal traités et par la RO, ils ont contribué à démontrer l'efficacité de la nouvelle approche. Ils ont également concouru à l'institutionnalisation en insérant l'enseignement des SAA dans certains curricula.

\section{Le Ministère de la Santé}

L'appui des CAs s'est toujours fait sous la tutelle du Ministère de la Santé, en particulier le service chargé de la santé maternelle. Afin de faciliter la collaboration entre les différents acteurs, un comité technique ou un comité de pilotage était mis en place. Il était dirigé par un responsable du ministère. Les personnes composant ces instances étaient généralement considérées comme des acteurs clés dans le processus de sensibilisation et de mobilisation des autorités et des prestataires.

\section{Les initiatives privées et ONG}

Des investissements ont été faits par les structures hospitalières (Hôpital Principal de Dakar structure à caractère privé) ou des ONG (SanFam) pour l'organisation des services et l'achat du matériel. Des ONG comme le CEFOREP et le CRESAR ont joué un rôle actif dans la RO, et subséquemment dans l'institutionnalisation, la formation et l'extension des SAA.

\section{Passage à l'échelle des soins après avortement}

\section{Vision et plan}

Les premiers pays à avoir introduit les SAA (Burkina Faso et Sénégal) ont dû passer par une expérience pilote réalisée dans les structures de référence de niveau national, telles que les CHU et les hôpitaux nationaux. La recherche opérationnelle menée au Burkina Faso avait comme sites les deux CHU de Ouagadougou et de Bobo Dioulasso. Le Sénégal avait intégré, dans sa première recherche opérationnelle un $\mathrm{CHU}$, un hôpital national à caractère privé, et un centre de santé situé en milieu semi urbain, mais dont le volume de ses activités gynécologiques et obstétricales était comparable à celui des hôpitaux nationaux. Pour le Niger et la Guinée, bien que les promoteurs se soient inspirés des résultats de l'expérience des deux premiers pays, il a fallu démarrer les activités dans les grands centres hospitaliers, avant de penser à les étendre aux structures périphériques. Enfin, dans les pays tels que le Mali et le Togo, la logique d'expérience pilote à étendre dans d'autres sites n'a pas prévalu. Des structures de référence nationale de même que d'autres situées en zone décentralisée ont été incluses dès la phase d'introduction. Les démarches empruntées par les pays sont exposées dans ce qui suit.

Au Burkina Faso, selon un des interlocuteurs qui a participé au processus de mise en place des SAA dans ce pays, la décision d'étendre les SAA dans d'autres sites en cas de réussite de l'expérience pilote qui devait être menée dans les CHU de Ouagadougou et de Bobo Dioulasso avait été prise dès le début: "... dès le départ c'était prévu qu'on ferait l'expérience et, si c'était bien, nous allions étendre ça à d'autres centres." Un autre interlocuteur d'ajouter : "Notre vision c'était de couvrir, en fait sur le plan de soins après avortement..., de couvrir le Burkina pour l'offre de services. Et une autre vision c'était que ce ne soit pas des services verticaux mais intégrés dans les soins que nous offrons d'une façon générale." Avec les résultats probants obtenus lors de la RO, il a été plus facile de convaincre les autorités à adhérer à l'idée d'intégrer les SAA dans d'autres structures. Cependant, étant donné le caractère sensible de toute question 
ayant trait à l'avortement, il fallait reconduire le même processus de dialogue, de sensibilisation et de plaidoyer pour amener les populations et autorités locales à ne pas considérer l'initiative comme un moyen détourné de "...libéraliser l'avortement pour que les jeunes filles puissent avorter facilement...". Les nombreuses rencontres et actions réalisées en milieu décentralisé ont entraîné l'adhésion des populations, des autorités, ainsi que des professionnels de santé. Ce travail de sensibilisation et de plaidoyer a été fait par le CRESAR et la DSF. Mais les activités techniques ont été réalisées par le CRESAR : "...c'était beaucoup de travail, parce qu'il y avait des réunions de concertation. On devait aller voir les besoins sur place et faire le nécessaire pour équiper ces maternités pour la prévention des infections, puisque c'était vraiment la base, et pour le traitement." (Membre de la CRESAR, Burkina Faso)

La démarche globale a consisté à entamer le processus par le niveau le plus élevé de la pyramide sanitaire, pour ensuite, toucher les hôpitaux régionaux et les structures périphériques. Pour le personnel, les formateurs du CHU ont formé en SAA le personnel des structures périphériques, charge à lui de former les autres membres du personnel. "...on a formé les gens des différents CHR puis on les a épaulés pour aller former les gens dans leurs différentes structures."

Cependant, le CHU, vu son statut, n'a pas pu étendre ses actions jusqu'au sein des communautés. "Au départ c 'était les trois éléments et puis après ça a été élargi à cinq, donc ce volet n'avait pas été pris en compte au début du programme."

Les projections faites par les promoteurs de l'époque n'ont pas trouvé d'appui, dans la mesure où, après l'introduction des SAA au Burkina Faso, l'on a observé un délai relativement long avant que les premières actions de mise à l'échelle fussent entamées. Pourtant un universitaire, membre de la CRESAR disait que : "On avait fait un plan de décentralisation, mais nous n'avons pas eu de suivi sur le plan des finances ; c'est ce qui nous a amené à interrompre le processus."

Par contre, pour un interlocuteur membre d'une institution partenaire, l'on ne pouvait pas parler de plan prévisionnel pour le passage à l'échelle des SAA. Selon lui : "Il n'y a pas de plan prévisionnel, nous avons pour objectif de couvrir l'ensemble des formations sanitaires de notre zone en soins obstétricaux d'urgence en tenant compte des soins après avortement". Cette information n'a pu être confirmée par le personnel de la DSF interrogé sur la question. Ceci révèle un manque de communication entre les différents acteurs et ramène la question du leadership, qui, selon un interlocuteur, a fait défaut.

Au Sénégal, le passage à l'échelle avait été décidé par le Ministère de la Santé, dès la publication des résultats de la recherche opérationnelle. En fait, le plan d'extension des SAA avait été élaboré lors de l'atelier d'élaboration des Politiques, Normes et Protocoles en SAA en 1998, avec l'appui du Population Council et de JHPIEGO. A l'époque, il avait été décidé d'introduire les SAA dans un certain nombre de structures sanitaires en milieu décentralisé et situées à différents niveaux de la pyramide sanitaire, en définissant le rôle et les prérogatives de chacune d'elles. D'un point de vue formel, il s'agissait d'introduire les SAA dans les hôpitaux régionaux, les centres de santé de district, les postes de santé et en milieu communautaire. Cependant, l'usage de l'AMIU n'était accepté que dans les hôpitaux et les centres de santé. Dans les postes de santé, il avait été proposé de former le personnel, composé d'infirmiers, avec parfois des sages-femmes et/ou des agents de sanitaires, à la détection des signes d'avortement ou de complication d'avortement, au counseling PF, aux gestes d'urgence pour la préparation de la référence et au curage digital. 
L'introduction des SAA en milieu communautaire avait été planifiée, mais dans une optique d'expérimentation, à cause du manque de données sur sa faisabilité et son efficience.

Cette volonté de généraliser l'utilisation du nouveau modèle de SAA sur l'étendue du territoire national était, cependant, tempérée par le fait que les moyens financiers provenaient exclusivement d'organismes partenaires qui ne pouvaient s'investir que dans un certain nombre de régions ou districts. Par ailleurs, étant donné le caractère sensible du sujet, les promoteurs, sous le leadership de la Division de la Santé de la Reproduction (DSR) avaient le souci d'éviter toute dérive qui serait préjudiciable à l'avenir du programme. C'est ainsi qu'il avait été décidé de choisir les premières structures à recevoir les SAA, en fonction des qualités présumées des responsables et des autres personnels des maternités ayant le potentiel technique requis.

La première phase du passage à l'échelle a concerné 5 structures sanitaires situées dans 5 régions, sur les 10 que comptait le pays à l'époque, avec l'appui de JHPIEGO. Il s'agissait de 4 hôpitaux régionaux (Diourbel, Saint-Louis, Kaolack, Ziguinchor) et d'un centre de santé situé dans une région où l'hôpital n'était pas encore fonctionnel (District de Sokone dans la région de Fatick). Les résultats satisfaisants obtenus surtout, dans le centre de santé de Sokone, a encouragé les autorités à poursuivre l'initiative au niveau opérationnel. C'est ainsi, qu'avec l'appui de Engender Health, l'introduction s'est poursuivie dans les centres de santé et les postes de santé de deux régions où le modèle était déjà utilisé (Kaolack et Fatick). Par la suite, toujours sous le leadership de la DSR, Intrah Prime a expérimenté le modèle SAA en milieu communautaire, plus précisément dans le district sanitaire de Sokone.

Ce passage à l'échelle avait été documenté par des activités de recherche qui ont permis de mettre à la disposition des décideurs les informations nécessaires pour la réussite de l'initiative. Ces recherches, dont les parties prenantes étaient la DSR, Population Council, le CEFOREP, l'UNFPA, Engender Health et Intrah Prime, ont montré l'efficience et la souplesse d'utilisation du modèle de SAA, car il a été démontré qu'il pouvait être adapté, avec plusieurs avantages, à tous les niveaux de la pyramide sanitaire, tout en gardant une cohérence d'ensemble.

A la suite de ces premières phases d'extension, JHPIEGO a touché deux nouvelles régions (Thiès et Louga), mais uniquement les hôpitaux régionaux. MSH a pris le relais en introduisant les SAA dans 23 centres de santé et 300 postes de santé de 5 régions cibles (Thiès, Louga, Ziguinchor, Fatick, Kaolack). Dans la même période l'UNFPA introduisait les SAA dans deux régions situées au Sud et au Sud-est du pays (Kolda et Tambacounda).

En guinée, selon un responsable du Ministère de la Santé, la vision du passage à l'échelle était de rapprocher les services des populations. La démarche était, qu'après avoir fait dans les hôpitaux nationaux, il fallait intégrer les hôpitaux régionaux, les hôpitaux préfectoraux et puis aller vers les centres de santé, selon un acteur de l'introduction des SAA dans ce pays. Selon lui, en fait cette démarche avait été dictée par un besoin d'éviter les risques de dérive qui seraient très importants si une politique massive de passage à l'échelle avait été menée "...on s'est dit qu'il faut canaliser les hôpitaux avant d'aller vers les centres de santé...". Il poursuit : "...selon le plan d'intégration qui est dans le plan stratégique de la santé de la reproduction on devait couvrir tous les hôpitaux, puis aller vers les centres de santé." Le plan prévisionnel a été défini par le ministère de la santé en collaboration avec les bailleurs de fonds, dans le cadre du plan stratégique de la SR 2000-2015. 
Selon un formateur, responsable au ministère de la santé, après l'adoption de la stratégie, la première unité a été installée à l'hôpital national de Donka. Une année après (2003), une évaluation a permis d'installer la seconde unité (hôpital de Niassa). Ensuite, l'appui de PRISME et JHPIEGO a permis l'extension vers d'autres sites. Dans ce cadre, il y a eu un échange d'experts entre la Guinée, le Sénégal et le Burkina pour les visites de suivi. Ces échanges se sont faits dans une collaboration très étroite.

Cependant, a noté un autre agent du Ministère de la Santé, l'ensemble du pays n'est pas couvert, mais tous les hôpitaux régionaux le sont. D'ici 2010, tous les hôpitaux préfectoraux seront couverts et les centres de santé d'ici 2015. D’ici 2008, les SAA seront intégrés dans les 2 grandes cliniques privées de Conakry.

Le besoin de former d'abord le personnel des hôpitaux était justifié par le souci d'avoir une bonne cohérence dans la gestion du processus à l'intérieur des régions. Ainsi, les personnels des hôpitaux déjà formés devaient ensuite superviser ceux des centres de santé. Cette volonté de cohérence était aussi doublée d'un souci d'adopter une approche à moindre coût. Pour ce faire, des recherches ont été initiées pour évaluer les actions menées. Cela a permis de faire le point de la situation à chaque étape, afin d'apporter des corrections puis de poursuivre l'extension.

Au Mali, selon un responsable de structure sanitaire, la vision qui sous-tend l'extension de la stratégie des SAA est d'apporter des solutions efficaces aux souffrances des femmes. "...la vision qu'on a c'est vraiment le soulagement des femmes", a-t-il déclaré. En pratique, il s'agira de procéder à la formation de formateurs nationaux et régionaux (2 par région), puis à la formation des prestataires dans les régions.

Seulement, le Ministère entend faire jouer à chaque région un rôle primordial dans la définition de ses propres priorités et des moyens à mobiliser pour les réaliser. Cela, en intégrant les activités de formation, d'approvisionnement et de supervision dans les PO des instances locales. Ce travail sera fait sous la tutelle des directions régionales de la SR.

Toujours selon cet interlocuteur, la décentralisation descendra jusqu'au niveau CSCOM. Il ajoute : "Pour le moment c'est prévu [pour] les hôpitaux régionaux, les centres de santé de référence, parce que à Bamako on peut aller jusqu'aux centres de santé communautaires qui sont tenus par des médecins et des sages-femmes, comme les centres de santé de référence. Mais pour le moment, ils ne font pas d'activités chirurgicales, mais il n'est d'abord pas question de dépasser les centres de santé communautaires, ça s'arrête au niveau de la référence." En fait le passage à l'échelle est accompagné d'une responsabilisation pleine des structures locales. En effet, selon un responsable de la DSR : "...si on fait d'abord la formation des formateurs deux par région, après, avec la décentralisation, les régionaux, [...] c'est à eux de faire la formation de leurs formateurs et aussi d'étendre à leur niveau. Nous, au niveau national, on va étendre, mais au niveau régional, ce sont les régions qui vont étendre à ces centres. Mais toujours en respectant la dotation des kits dans les PO, en intégrant la formation dans les PO."

La décision de privilégier les initiatives locales, notamment les régions, pourrait être à l'origine de l'absence d'échéances claires au sujet de la mise à l'échelle au Mali. En effet, les principales autorités interrogées ont déclaré qu'il reviendra aux régions et les structures qui dépendent de leur juridiction de trouver les voies et moyens pour assurer l'implantation des activités de SAA. La question est de savoir si, avec cette approche, la DSR pourra jouer un rôle moteur pour 
amener les instances locales à investir de manière conséquente en faveur du développement des activités SAA.

Le secteur privé de la santé n'est pas oublié dans le développement de la SR. Selon un responsable à la DSR : "...nous impliquons les privés. Ils sont organisés en association. On demande, par exemple, à l'association d'envoyer deux structures pour telle formation, [...] soit ils viennent, soit ils ne viennent pas. Mais quand même ils sont impliqués de façon officielle dans toutes les formations continues." (Responsable au Ministère de la santé, Mali). Néanmoins, pour ce qui concerne les SAA, les cliniques privées n'ont pas encore été associées aux activités de formation. Les responsables interrogés ont quand même annoncé que ces structures sanitaires seront impliquées plus tard. Cependant, un interlocuteur universitaire au Mali a émis des réserves sur la capacité du secteur sanitaire privé à développer une politique de SAA. Selon lui : "...je ne pense pas que le secteur privé soit structuré globalement de façon conséquente pour vraiment une politique de SAA suffisamment efficace." Le volet communautaire n'est pas encore prévu dans le processus de mise à l'échelle.

Au Niger, après la formation du premier groupe de formateurs, tous issus de la maternité I. Gazobi, les activités de SAA ont démarré immédiatement dans cette structure.

Le besoin s'est fait sentir d'étendre la pratique des SAA à l'intérieur du pays, suite aux prospections effectuées par l'ONG FCI dans plusieurs régions du pays. C'est ainsi que, grâce à un important appui matériel et financier de cette organisation partenaire que l'équipe de la maternité I. Gazobi a commencé à former les prestataires des structures sanitaires de la région de Zinder. Par la suite, la région d'Agadez a reçu l'équipe de formateurs.

Un plan prévisionnel d'extension des SAA a été élaboré en collaboration avec les organismes partenaires en santé, dont la Coopération Belge en est le chef de file. Par ailleurs, grâce au plaidoyer mené par le médecin chef de la maternité I. Gazobi et la DSR, la participation de l'UNFPA au processus d'extension des SAA sera effective dans les régions de Niamey et de Thilabéry, tandis que la Banque Mondiale couvrira les régions de Dosso et de Tawa.

\section{Niveaux atteints dans la décentralisation ou la mise à l'échelle}

Tous les pays visités ont dépassé l'étape d'introduction des SAA, bien que le Mali et le Togo n'aient démarré leurs activités que récemment. Dans ces deux derniers pays, la stratégie d'introduction comprenait, outre les structures de référence nationale, mais aussi quelques infrastructures situées dans les régions. L'on peut considérer que pour ces pays, il s'est plutôt agi d'une décentralisation des SAA.

Pour les pays pionniers dans le domaine des SAA (Burkina Faso, Sénégal), le passage à l'échelle a permis de couvrir l'ensemble des structures de référence régionale (l'équivalent des hôpitaux régionaux). Au Burkina Faso, il s'agissait de 13 régions, tandis qu'au Sénégal, on comptait 11 régions. Néanmoins, il reste encore un certain nombre de districts sanitaires qui n'offrent pas de SAA. La répartition des districts sanitaires couverts recoupe globalement les zones d'intervention des organismes partenaires. Au Sénégal, par exemple, entre 2003 et 2006, 23 centres de santé et 300 postes de santé ont bénéficié d'un appui de MSH, pour former leurs personnels, réorganiser leurs services et prendre en charge les femmes souffrant de complications d'avortement. Les structures concernées étaient situées dans les régions traditionnellement ciblées par l'USAID que sont Thiès, Louga, Kaolack, Fatick et Ziguinchor. Au terme du programme, tous les centres de 
santé et $72 \%$ des postes de santé ont été touchés par les interventions. Cet appui a été réalisé dans le cadre de l'accord bilatéral entre le Gouvernement sénégalais et l'USAID. Dans la même période, l'UNPA menait des interventions dans les régions de Kolda et Tambacounda dans les hôpitaux régionaux et les 8 centres de santé de district, de même que dans un nombre important de postes de santé. En Guinée, par contre, tous les hôpitaux préfectoraux ne sont pas encore couverts. Selon un interlocuteur, il en resterait une dizaine à toucher.

Pour ce qui concerne le secteur privé, si le Mali entend l'associer officiellement dans le processus de passage à l'échelle, il n'en est pas encore de même dans les autres pays, que cela soit en Guinée, au Burkina Faso, au Sénégal au Niger et au Togo.

\section{Encadré 1 : Activités de passage à l'échelle des SAA réalisées par MSH au Sénégal}

- La Division de la Santé de la Reproduction du MS est très favorable à l'introduction des services de SAA de qualité dans les structures du niveau primaire.

- Dans les centres de santé, le nombre de patientes traitées pour des complications après avortement a augmenté de 1178 en 2003 à 2530 en 2005. Parmi celles-ci, 53\% ont été traitées grâce à l'AMIU.

- Le pourcentage de patientes ayant reçu un counseling de PF après traitement est passé de $36 \%$ en 2003 à $78 \%$ en 2005.

- $56 \%$ des patientes ayant reçu un counseling sont retournées chez elles avec une méthode contraceptive

- Grâce à la supervision, les services ont été réorganisés dans les 23 centres de santé.
- Manque d'appropriation des services de SAA au niveau du poste; la plupart des patientes sont immédiatement référées vers un niveau supérieur sans aucun soin.

- Manque de référence vers d'autres services de SR pour satisfaire les besoins des femmes

- Manque de liaison avec les services communautaires

- Remplissage incomplet des registres de SAA

- Indisponibilité des méthodes de planning familial dans le site où les services d'urgence sont offerts

- Manque de supervision par le MS dans 20 districts sur les 23 après la réduction des activités par MSH. Une assistance externe pour le transport était nécessaire à la bonne organisation de la supervision

- Les hôpitaux régionaux n'ont pas participé à cette phase, et ceci n'a pas été très apprécié par les prestataires de ces structures de santé.

Source: Document Ponctuel \#5, 2006

\section{Zones non couvertes par les bailleurs}

Comme la plupart des programmes de santé, les SAA sont tributaires de l'appui que les pays, ou les institutions partenaires leur accordent. Cependant ils limitent leurs interventions à quelques régions et districts. De ce fait, certaines zones non couvertes restent vierges de toute activité majeure. C'est le cas dans tous les pays, plus particulièrement dans les premiers à avoir introduit les SAA (Burkina Faso, Sénégal, Guinée, y compris Niger).

Face à ce problème, des pays comme le Burkina Faso et le Sénégal, par exemple, on signé un accord de partenariat avec IPAS, pour la formation des personnels de santé de quelques régions non encore couvertes, et un approvisionnement en kits AMIU par cette même structure. Au Sénégal, cette collaboration s'est concrétisée avec la formation de sages-femmes de la région de Dakar, et de formateurs de l'Ecole Nationale de Développement Sanitaire et Social d'où sortent les sages-femmes. Le Burkina Faso a planifié de construire les hôpitaux de référence des deux régions qui n'en disposent pas encore. D'après un conseiller au Bureau local de l'OMS, ce pays projette d'étendre les SAA dans toutes les structures habilitées (hôpitaux et CMA), dans le cadre 
d'un programme global qui devrait mobiliser l'ensemble des partenaires. En Guinée, selon un interlocuteur, les 10 hôpitaux préfectoraux restant seront certainement pris en compte dans le Programme National de Développement Sanitaire (PNDS), financé par la Banque Mondiale, car ils sont prioritaires à cause de leur éloignement et de leur enclavement.

\section{Institutionnalisation des soins après avortement}

\section{Elaboration, mise en œuvre et mise à jour des Politiques, Normes et Protocoles en SAA}

Entre 1998 et 2005, dans tous les pays visités, les SAA ont été intégrés dans les documents des Politiques, Normes et Protocoles. A l'heure actuelle, dans certains pays, ils ont été réactualisés avec à un modèle à 5 composantes (Sénégal, Burkina Faso, Guinée). Dans d'autres pays, par contre, c'est le modèle à 3 composantes qui a officiellement cours (Togo, Niger). Les processus de révision devraient être entamés incessamment. Au Mali, où l'intégration a été tardive (2005), c'est le modèle à 5 composantes qui a été directement introduit. Ces ouvrages sont issus du processus de standardisation initié par AWARE-RH.

\section{Intégration des SAA dans les curricula de formation continue}

Le module de formation en SAA, issu du processus de standardisation initié par AWARE- RH, a été intégré dans les curricula de formation continue des pays visités. Il a été introduit par le CEFOREP, dans la sous-région, au cours des formations de prestataires ou de formateurs. En fonction des besoins en formation et du contexte de chaque pays, ce module de base a été adapté par le Ministère de la santé et les formateurs nationaux. Ainsi, au Burkina Faso, l'accent est mis sur le counseling, la prévention des infections, les liens avec les services de la reproduction et la pratique de l'AMIU. En Guinée, il a été intégré dans un paquet d'activités exécutées dans un ordre chronologique : formation en prévention des infections, formation en technologie contraceptive, la visite de préparation, puis formation en soins après avortement proprement dite ; au Sénégal, il a été adapté à 3 types de prestataires (sages-femmes, infirmiers chefs de poste et conseillères en $\mathrm{PF}$ ).

Au Mali, au Togo et au Niger, dans le cadre des activités de AWARE-RH et dans le processus de mise à l'échelle, c'est le module introduit par le CEFOREP qui est utilisé.

Par ailleurs, il faut signaler qu'au Mali, il existe un autre outil de formation qui a été mis à la disposition du CHU par IPAS pour les formations que cette institution finance.

\section{Intégration des SAA dans les curricula de formation de base}

En fonction des pays et du profil de l'apprenant, le degré d'intégration des SAA dans les curricula de formation de base est variable.

A l'université, pour les étudiants en $5^{\text {ème }}$ année de médecine ou $7^{\text {ème }}$ année (Niger), des initiatives sont en cours de préparation au niveau des facultés de médecine avec la réforme des programmes. Pour l'essentiel des pays, les SAA ne sont pas enseignés de façon modulaire. Dans les faits, il s'agit plus d'une initiation que d'une réelle formation en vue de l'acquisition de compétences du fait de l'importance des effectifs (cas du Burkina, du Sénégal ou du Mali). Elle se fait au cours des stages hospitaliers pratiques (cas de la Guinée et du Sénégal).

Dans les écoles de formations paramédicales, les élèves sages-femmes sont les plus concernées. Les élèves infirmiers aussi bénéficient d'une formation en SAA au Burkina Faso. Cette 
intégration ne concerne là aussi que les aspects théoriques. Les objectifs d'une formation pratique effective sont plus difficiles à atteindre du fait, là encore, de l'importance des effectifs à former (Burkina, Guinée et Togo). Au Niger, elle est envisagée pour l'année académique 20072008. Au Sénégal, le processus n'est pas encore entamé.

\section{Intégration des SAA dans les Plans Opérationnels}

Concernant ce point de l'institutionnalisation des SAA, on distingue deux groupes de pays. Dans le premier, comprenant les pays où l'implantation des SAA est relativement ancienne, (Burkina Faso, Guinée et Sénégal), les SAA sont pris en compte dans les plans annuels des districts. Au Burkina et au Niger, par exemple, les partenaires au développement appuient les structures décentralisées dans la formation et le renouvellement du matériel. Au Sénégal, par contre, ce sont les districts qui achètent, sur fonds propres, les kits AMIU à remplacer. En Guinée, les SAA sont intégrés dans la Feuille de Route et, à ce titre, ils figurent dans les plans annuels de chaque structure sanitaire.

Dans le second groupe de pays (Mali, Togo et Niger), le processus d'implantation et de mise à l'échelle des SAA est encore trop récent pour permettre leur intégration dans les plans des structures décentralisées.

\section{Système d'Information Sanitaire}

Les données relatives aux SAA sont systématiquement enregistrées dans l'ensemble des structures visitées. Dans les pays où les SAA ont été implantés depuis près de 10 ans, des registres standardisés et imprimés, réservés exclusivement aux activités de SAA sont utilisés (Sénégal, Burkina et Guinée). Dans les 3 autres pays, il s'agit de registres confectionnés selon les instructions des formateurs. Par exemple à la maternité Issaka Gazobi de Niamey, les registres ont été proposés par les formateurs du CHU Yalgado Burkina Faso, tandis que pour le Mali et le Togo, les registres sont inspirés de ceux proposés par le CEFOREP. En plus de ces registres, les $\mathrm{CHU}$, disposent de dossiers individuels pour les patientes. Le registre permet d'avoir une vision d'ensemble sur l'évolution des activités de SAA. Ces informations représentent une base de données pour la recherche car, après analyse, elles sont utilisées souvent par les étudiants pour leurs thèses (Burkina Faso, Mali, Sénégal). Cependant, dans certains sites, la compilation des données fournies par les différentes unités où les femmes sont prises en charge pour des complications d'avortement pose problème, surtout pour les activités des équipes de garde. Soit la compilation ne se fait pas de manière systématique, soit elle ne se fait pas du tout. Toujours est-il que dans plusieurs sites il n'existe pas réellement de système de collecte codifié et institutionnalisé ; la fiabilité du système repose sur la volonté d'une ou de quelques personnes. Par exemple, la responsable de l'unité de SAA d'une maternité à Niamey a affirmé qu'elle effectue, chaque matin, un tour à la salle d'accouchement, afin de noter les cas de complication d'avortement traités par la dernière équipe de garde. Elle les reporte ensuite dans le registre de SAA. Même si c'est une bonne initiative, l'on peut néanmoins s'interroger sur sa pérennité et sur le niveau de détail des données collectées dans la salle d'accouchement.

Il faut également souligner la faiblesse du système de référence et contre référence dans les 6 pays. Il est rare que la rétro-information après traitement se fasse de manière systématique et institutionnalisée. L'utilisation des fiches de contre référence est pratiquement inexistante. 


\section{Supervision}

L'un des problèmes majeurs que rencontrent les pays dans le processus d'extension des SAA, se trouve dans leur incapacité à assurer un système de supervisions régulières et suffisamment approfondies sur les questions relatives à la pratique des SAA. Cela est apparu dans les propos de tous les répondants, quel que soit le pays. Les difficultés qui sont à l'origine de ce problème sont liées à l'insuffisance de ressources humaines compétentes dans ce domaine. Ce sont généralement les pools de formateurs qui, en plus de leur travail au quotidien, doivent trouver du temps pour assurer la supervision des agents formés ; ceci, sachant que l'on parle le plus souvent de supervision formative. L'autre difficulté est relative à la périodicité des supervisions. Après les visites de suivi post formation, les agents peuvent rester pendant six mois ou plus sans recevoir la visite d'une équipe de supervision. De plus, les missions de supervision sont intégrées. Il n'y a pas, à priori de supervision spécifique pour les SAA ; ce sont l'ensemble des volets de la santé de la reproduction (SR) qui sont passés en revue. Dans certaines situations, la SR n'est qu'un élément de cette supervision. Dans ce dernier cas de figure, comme cela se passe souvent ailleurs, les supervisions sont réalisées en fonction de l'actualité. Ainsi, une mission de supervision peut ne s'intéresser qu'à la prévention et la prise en charge des cas de méningite. Cela a souvent comme conséquence la tenue de supervisions qui ne font qu'effleurer les problèmes et dont les rapports ne fournissent que des recommandations générales. Selon un répondant au Burkina Faso "...au niveau national, on préconise la supervision intégrée, ça veut dire que quand les équipes se déplacent sur le terrain, elles doivent voir pratiquement toutes les prestations, et cela parfois joue en défaveur de la qualité et principalement dans certains domaines." Il poursuit "...on se rend compte que, tout au plus, on a pu s'intéresser aux aspects de la SR, peut être une fois au cours de l'année, et encore de quelle manière ?"

Il faut souligner que la Guinée dispose d'un système de supervision régulier, parallèle, spécifique aux SAA, mais dont la pérennité reste suspendue aux financements des partenaires au développement.

Par ailleurs, au Sénégal comme au Togo, les superviseurs régionaux n'ont pas une vision claire du modèle de SAA, contrairement aux prestataires. Cela pose le problème de leur implication dans la formation des prestataires (supervision formative) alors qu'ils ne pratiquent souvent pas les SAA, ou alors de la nécessité de les orienter afin qu'ils puissent remplir leurs fonctions avec des supervisions facilitatrices.

\section{Formation des prestataires}

\section{Politique de formation}

Cette thématique est un aspect clé du développement des SAA. Cependant, il est généralement difficile, pour les responsables, de parler de politique de formation en SAA de manière formelle, dans la mesure où, selon eux, il n'existe pas un document écrit à cet effet. Les activités de formation sont le plus souvent planifiées et menées en fonction des programmes proposés par les organismes partenaires, bien qu'il existe une volonté affichée de former le maximum de prestataires.

La dualité qui existe entre la formation continue et la formation de base en SAA se pose pour certains responsables en termes d'équilibrage. La formation continue s'impose en raison du besoin de mettre à jour les connaissances et compétences des prestataires déjà en service, avec l'avènement de nouvelles approches thérapeutiques. "...nous sommes obligés de faire la 
formation continue, mais il est important que la formation continue ne prenne pas le pas sur la formation de base." (Chargé de programme DSF, Burkina-Faso)

Un organisme partenaire propose au Burkina Faso une politique dont l'un des objectifs est la formation des prestataires dans les 13 régions du pays. Cette politique consiste à constituer une équipe de formateurs au niveau central. Cette équipe se chargera de mettre en place et de superviser les équipes régionales de formateurs qui se chargeront de développer les compétences des prestataires dans leurs circonscriptions respectives. Des politiques similaires sont déjà appliquées en Guinée et vont l'être au Mali, selon certains interlocuteurs.

Il faut cependant préciser qu'en Guinée, la question de l'existence d'une politique de formation des ressources humaines en SAA n'est pas comprise de la même façon par tous les répondants. En effet, pour d'aucuns ce système existe avec des formateurs nationaux et régionaux, tandis que pour d'autres, ce qui est fait relève plutôt d'initiatives personnelles, sans une véritable articulation entre les différents intervenants. Toutefois, l'un des tenants de la thèse de l'existence d'une politique générale de formation intégrant les SAA insiste et annonce que la révision de celle-ci est en cours. La formation des prestataires concerne ceux en service dans les hôpitaux nationaux, les hôpitaux régionaux et les centres de santé préfectoraux.

Au Mali et au Togo, les activités de formation en sont à leur début. Selon un répondant au Mali, la mise à l'échelle des SAA touchera toute la pyramide sanitaire, du niveau de référence national aux Centres de Santé Communautaires (CSCOM) disposant de médecins. Jusqu'à présent, seuls les médecins et les sages-femmes bénéficiaient de la formation en SAA. Il faudra, selon lui, inclure le corps des infirmiers-accoucheurs, si l'on veut réussir la mise à l'échelle jusqu'au niveau communautaire. A l'heure actuelle, cette politique de formation dispose d'une planification nationale gérée par la DSR.

\section{Formation continue}

\section{Formation en atelier}

De 1997 à l'heure actuelle, c'est la principale méthode de formation des prestataires en SAA. La principale raison de cette situation est liée au fait que les formations sont le plus souvent initiées dans le cadre de programmes qui sont obligés d'intégrer dans leurs activités la formation des prestataires déjà en service, afin d'atteindre les objectifs qui leur sont assignés en quelques années. Cette logique qui pouvait se justifier lors des premières initiatives d'introduction des SAA, dont l'objectif principal était de démontrer l'efficience de la nouvelle approche, ne semble plus répondre au contexte actuel. D'ailleurs, de plus en plus, les SAA sont considérés comme un volet de programmes plus globaux comme les Soins Obstétricaux d'Urgence, même si on continue à mener des formations spécifiques en SAA. D'après plusieurs interlocuteurs, les formations continues organisées sous forme de séminaires/ateliers sont coûteuses et réduisent souvent la disponibilité du personnel, le temps d'une session, pour ce qui concerne l'offre de services, particulièrement dans les zones périphériques qui souffrent d'un manque de personnel.

La situation de dépendance envers les bailleurs de fonds non étatiques est encore perçue comme une gêne sur fonds d'inquiétude à propos de l'efficacité et de la pérennité de la stratégie de renforcement des capacités des ressources humaines par le biais de la formation continue. 


\section{Pool de formateurs}

Dans les premiers pays qui ont introduit les SAA, un pool de formateurs nationaux a été d'abord mis en place. Les membres de ces équipes étaient généralement des gynécologues et étaient en service dans les sites où les expériences pilotes ont été réalisées. Ce fut le cas au Burkina Faso, au Sénégal, en Guinée, de même qu'au Niger. Les pays qui sont venus dans un second temps (comme le Tchad, le Mali, le Cap-Vert, la Guinée Bissau et le Togo) ont, quant à eux, dès le démarrage de leur programme, créé une équipe de formateurs composée de personnes en position au niveau des hôpitaux de référence, mais aussi d'autres agents provenant de structures décentralisées. Le principal enjeu de certains responsables, pour la formation continue, est de créer des équipes de formateurs au niveau de chaque région. Selon eux, cela permettra de réduire les coûts des formations, de réduire les périodes d'absence des personnels pour les besoins de formation et, en même temps, d'assurer une supervision plus régulière des prestations de service. Dans les pays visités durant l'étude, les pools de formateurs comportaient des médecins, ainsi que des sages-femmes. Dans la majeure partie des cas, il s'agissait de formateurs au niveau national. Dix formateurs composent l'équipe du Burkina Faso, 12 en Guinée (dont 7 dans les régions) et 8 au Niger. Au Sénégal, depuis l'introduction des SAA, plusieurs équipes de formateurs ont été créées, surtout dans les régions où l'USAID et l'UNFPA s'investissent. Dans ces zones, ce sont généralement des membres de l'équipe de district qui procèdent à la formation et à la supervision des prestataires de leur zone. Au Togo, il y avait 3 équipes de formateurs, dont 2 dans des villes secondaires. Au Mali, les avis semblaient divergents sur l'existence d'un pool de formateurs. D'aucuns estimaient le nombre de formateurs à 12, tandis que d'autres ne considéraient que les deux agents qui ont reçu la formation de formateurs à Dakar ; les autres bénéficiant d'un système de compagnonnage, n'avaient pas, à leurs yeux, un statut de formateur à proprement parler.

Tableau 2 : Nombre de formateurs en SAA selon les 6 pays de l'évaluation.

\begin{tabular}{lc}
\hline \hline Pays & $\begin{array}{c}\text { Nombre de } \\
\text { formateurs }\end{array}$ \\
Burkina Faso & 10 \\
Sénégal & 19 \\
Guinée & 12 \\
Mali & 2 \\
Niger & 8 \\
Togo & 10 \\
\hline \hline
\end{tabular}

Les équipes de formateurs régionaux étaient souvent sujettes à des déperditions assez importantes. Ce fut le cas de l'équipe de formateurs de Family Care International au Burkina Faso, dont deux des trois qui la composaient ont quitté la zone d'intervention. L'UNFPA déplore le même problème et cherche plutôt à renouveler les équipes de formateurs afin de résorber le déficit de personnels à ce niveau.

"...il y a une déperdition des formateurs; y en a qui vont faire santé publique ; y en a qui quittent et qui vont vers d'autres spécialités. Donc on perd beaucoup, ce ne sont que les gynécologues qui restent puisqu'ils sont déjà formés comme gynécologues que nous pouvons utiliser comme formateurs" (un responsable de structure, Burkina Faso).

En Guinée, les équipes de formateurs sont composées de médecins et de sages-femmes. Cependant, les déperditions sont plus importantes chez ces dernières, essentiellement à cause de leur charge de travail importante. 


\section{Les prestataires à former}

Les agents ciblés pour être formés en SAA sont généralement les médecins gynécologues, ainsi que les sages-femmes. Le choix de ces deux catégories de personnel se justifie par leurs compétences en matière de gynécologie et d'obstétrique. En les responsabilisant, on réduit ainsi le temps de formation, et on s'assure en même temps que les complications peuvent être prévenues, ou bien identifiées précocement et prises en charge dans le cas échéant. Cependant, dans le contexte d'un passage à l'échelle, le manque de personnels ayant ces deux profils peut pousser les autorités à élargir les possibilités en y incluant des infirmiers et médecins ayant acquis quelques compétences en soins obstétricaux d'urgence. C'est le cas au Sénégal, où des médecins appelés "Compétents SONU", de même que des infirmiers ont reçu une formation en SAA. Dans certaines régions, les infirmiers chefs de poste de santé ont été formés à tous les volets du paquet de services qu'offrent les SAA, sauf la manipulation de la seringue, dont l'utilisation se limite aux hôpitaux et aux centres de santé (au Sénégal, jusqu'à présent, seules les structures ayant des médecins peuvent pratiquer l'AMIU). Ces infirmiers peuvent détecter les signes de complications d'avortement, faire le counseling, faire le curage digital si les conditions s'y prêtent, offrir une méthode contraceptive et référer les complications plus sévères vers des structures de référence. Au Mali, la DSR envisage de former en SAA les infirmiers-accoucheurs pour les Centres de Santé Communautaires disposant de médecins, mais n'entend pas inclure les infirmiers simples dans cette formation.

\section{Efficacité et pérennité de la formation continue}

La question de l'efficacité et de la pérennité de la formation continue, dans sa formule actuelle, se pose davantage dans le contexte de décentralisation/passage à l'échelle. En effet, au niveau périphérique on peut identifier trois problèmes qui ont un impact non négligeable sur l'efficacité de la stratégie de formation continue des prestataires : les coûts de formation qui sont généralement entièrement supportés par les bailleurs ; la mobilité du personnel qui fait qu'on est obligé de reprendre la formation chaque fois qu'il y a un personnel nouveau et le suivi de ce personnel pour assurer le maintien de la qualité des services à un bon niveau.

D'autres problèmes apparaissent très souvent dans le cadre de la formation en milieu décentralisé. D'après certains interlocuteurs, les stagiaires n'ont souvent pas la possibilité de pratiquer suffisamment de cas, à cause de la faible fréquentation des structures périphériques. Ce fait a surtout été relevé au Sénégal et au Niger. Pour pallier cette insuffisance, l'exercice sur les mannequins est considéré comme une méthode de rechange ayant une certaine efficacité. Cependant, ces outils aussi font défaut, surtout en milieu décentralisé, comme cela a été souligné au Niger.

\section{Formation par les pairs}

Face à l'insuffisance des ressources pour organiser des séminaires de formation en SAA, plusieurs structures ont initié des tentatives de formation de leurs personnels avec l'aide de ceux d'entre eux qui l'étaient déjà. Cependant, cette approche n'a pas été abordée par nos interlocuteurs du niveau central et par les bailleurs. Seuls les responsables des structures de santé et les prestataires en ont fait mention. L'attitude de ces responsables révèle une non prise en compte, dans le cadre des programmes et stratégies, du potentiel que peut receler ce type de formation, et de son effet possible sur la qualité des services.

Cette approche appelée communément "formation en cascade" permet, selon plusieurs interlocuteurs, de renforcer les capacités des prestataires à moindre coût. Ce type de formation 
cible aussi bien les sages-femmes en service, que les médecins en spécialisation en gynécologie obstétrique (CES), et il est dispensé par des médecins formateurs, mais encore par les sagesfemmes responsables des unités de SAA. La formation "sur le tas" ou formation non formelle peut revêtir plusieurs formes. En effet, au Burkina Faso, certains prestataires, surtout les CES, ont été intégrés dans des sessions de formations formelles animées par leurs enseignants au profit de personnels d'autres structures. Ils ont pu bénéficier de tous les aspects théoriques et pratiques qui sont dispensés lors des ateliers de formation en SAA. Ainsi, au CHU Yalgado de Ouagadougou, selon un prestataire formateur en SAA, environ soixante (60) agents ont été formés de façon formelle en profitant des sessions de formation dédiées à d'autres personnels. A côté de ce type de formation, d'autres prestataires ont été initiés aux SAA dans le cadre de leurs activités régulières. Ce type de formation est dispensé par des prestataires déjà formés, qu'ils aient acquis un statut de formateur ou non dans ce domaine. Généralement les prestataires en quête de compétences observent leurs collègues pratiquer sur un certain nombre de cas. Puis, ils s'exercent directement sur des patientes admises pour traitement de complications d'avortement. Par ce biais, des prestataires ont affirmé avoir formé plus de cent quarante (140) agents au CHU Yalgado de Ouagadougou, environ 1000 agents et étudiants en médecine au CMA du Secteur 30 de la même ville, depuis 2003. Dans cette structure, en dehors de l'équipe initiale de sagesfemmes qui avait été formée au CHU, toutes les autres ont été initiées "sur le tas". Cette formation a été organisée selon le plan suivant. Les candidates étaient recrutées parmi les sagesfemmes qui étaient de service au niveau de l'unité des suites de couches. Chaque mois, l'une d'entre elles travaillait dans l'unité de SAA avec la sage-femme responsable. Elle passait en revue tous les aspects concernant le traitement et le counseling. Le choix de participer à la formation était libre. Cependant, au fil du temps, il y a eu de moins en moins de candidates, selon le médecin chef de la maternité.

Selon plusieurs prestataires, la formation par les pairs a donné des résultats positifs, dans la mesure où, celle-ci a permis de disposer d'un personnel compétent plus nombreux, surtout pour les équipes de garde. Dans l'ensemble des structures visitées, les prestataires ont affirmé que la formation non formelle transmise a augmenté l'offre de service SAA. Ainsi, dans certaines structures, les SAA ont pu se faire $24 \mathrm{~h} / 24$ grâce à cette approche. "...nous ne voyons plus traîner des patientes avec des feuilles de référence qui errent dans les structures à la recherche de ce service" disait un prestataire du Burkina Faso. Cette idée est confirmée au niveau du CHU de Yalgado par un prestataire en SAA qui souligne qu'il n'y a plus de "...cas en attente d'AMIU, toutes les femmes qui se présentent sont traitées par le personnel trouvé sur place. Ce n'est plus comme avant ; le temps d'attente est moins long pour les patientes."

Par contre, au Sénégal notamment, ce type de formation ne semble pas avoir donné les résultats escomptés. Dans plusieurs structures, les agents qui ont été encadrés par leurs pairs estiment que leur formation a été tronquée. De ce fait, ils refusent souvent de participer à la prise en charge des femmes admises pour des complications d'avortement incomplet, et préfèrent requérir le service d'un collègue ayant reçu la formation.

A ce manque de motivation noté dans certaines structures au Sénégal et au Burkina Faso, s'ajoutent les problèmes de locaux avec des unités de SAA qui se réduisent souvent en une salle ; ce qui pose énormément de problème en matière de pédagogie, sans parler du manque de matériel didactique. Cette situation réduit souvent la formation non formelle à la pratique de 
l'aspiration manuelle intra utérine, même si les autres composantes sont évoquées, mais de façon orale seulement.

Il est donc urgent que les institutions étatiques et de coopération, d'accorder une plus grande attention à ce type de formation. Ceci permettrait de l'évaluer et de proposer des améliorations qui pourraient autoriser des résultats plus importants.

L'une des démarches préconisées pour rendre plus efficace la formation continue est résumée par l'OMS dans le terme "Accompagnement". Selon un interlocuteur, l'on entend sous ce vocable un schéma de formation du personnel en service à l'échelle nationale, associée à une mise en place de tous les éléments nécessaires à un fonctionnement complet des services. Le tout réalisé selon une planification bien maîtrisée. Pour le volet développement des capacités des ressources humaines, la formation d'un noyau de formateurs nationaux en est la première étape. La formation des équipes de formateurs régionaux en constitue la seconde étape. Ainsi, à terme, les formateurs au niveau national joueront le rôle de formateurs et de superviseurs des formateurs régionaux. Le niveau central mettra donc en place un système d'accompagnement des régions et districts dans la mise en œuvre de la stratégie des SAA. Selon toujours cet interlocuteur, cette démarche permettra d'avoir plus vite des résultats tangibles.

\section{Formation à la base}

Les difficultés rencontrées par les responsables ministériels et par les dirigeants des programmes d'appui pour rendre disponible un personnel compétent en SAA, a poussé ces acteurs à initier des réflexions et des actions, qui visent à intégrer les SAA dans les curricula des institutions de formation des agents de santé ayant une spécialisation en gynécologie et obstétrique.

Cette question a été soulevée depuis un certain nombre d'années, notamment lors de la Conférence Africaine Francophone sur les SAA, en 2002. A l'époque, de nombreux acteurs avaient souligné l'urgence de voir les personnels de santé acquérir les compétences nécessaires à la pratique des SAA lors de leurs études médicales. Cependant, les responsables des structures de formation avaient rappelé les étapes nécessaires pour atteindre cet objectif. Selon le rapport analytique de cette rencontre, il est noté ce qui suit, au sujet de l'introduction des SAA dans les curricula des institutions de base. "Parce que ce processus de renforcement exige du temps, la formation continue est utilisée pour développer les compétences du prestataire, améliorer la performance et développer les sites de formation clinique. À mesure que les stagiaires sortant des institutions de formation de base viennent s'ajouter au pool national de prestataires, le besoin pour la formation continue baissera." Ce rapport mettait ensuite l'accent sur le gain en efficacité que l'on pourrait avoir en combinant la formation continue et la formation de base.

L'application des recommandations de la Conférence de 2002 a connu des fortunes diverses selon les pays. Pour la formation des agents de santé, hormis les CHU qui avaient déjà entamé la formation des CES et internes à la pratique des SAA, au Burkina Faso, au Sénégal, en Guinée et au Niger, les écoles de formation des étudiants en médecine, de même que les écoles de sagesfemmes n'ont pas atteint le même niveau d'avancement de l'intégration des SAA dans leurs curricula de formation d'un pays à un autre. En effet, si au Burkina Faso les élèves sages-femmes et infirmiers de l'Ecole Nationale de Santé Publique (ENSP) bénéficient d'un module sur les SAA, il n'en est pas de même au Sénégal, en Guinée, au Mali, au Niger et au Togo où cela est à l'état de projet plus ou moins avancé. Certains pays entendaient démarrer l'enseignement du nouveau modèle des SAA à compter de l'année académique 2007-2008. Il faut préciser qu'en 
fait, les SAA étaient enseignés dans les écoles de formation de sages-femmes et pour les étudiants en spécialisation en gynécologie, mais pas dans leur formule actuelle. Seul le curage digital et la dilatation et curetage étaient enseignés. La prévention des infections et le counseling n'étaient pas inclus dans l'enseignement. De plus, ces institutions ne disposent pas encore de formateurs en leur sein. Elles font recours aux formateurs des CHU.

Cependant, l'enthousiasme affiché par les responsables de l'ENSP du Burkina Faso, sur la qualité de l'enseignement des SAA, n'est pas toujours partagé par certains formateurs basés au CHU. Ces derniers mettent en exergue deux problèmes essentiels. Le premier est relatif à la qualité des stages pratiques qui n'est pas satisfaisante à leurs yeux, étant donné le nombre important d'élèves face à la capacité limitée des structures d'accueil. Ce qui fait que de nombreux élèves valident leur stage sans avoir pratiqué les SAA. Le second problème est lié au système d'évaluation. En fait, il n'y a pas d'évaluation portant spécifiquement sur les SAA. Les évaluations portent sur la gynécologie et l'obstétrique, et il arrive qu'aucune question ne se rapporte aux SAA. Pour ce qui concerne les facultés, le processus d'intégration semble encore plus long, et n'a pas connu de réelles avancées dans les pays.

C'est pour apporter une solution globale et définitive à ce problème, que l'Organisation Ouest Africaine pour la Santé (OOAS) a initié une action avec des universitaires de la sous région pour harmoniser les curricula de formation en Soins Obstétricaux et Néonataux d'Urgence et leur introduction dans les programmes d'enseignement des facultés de médecine et des écoles de formation de sages-femmes et d'infirmiers.

\section{Approvisionnement et équipement en matériels d'Aspiration Manuelle Intra Utérine}

\section{Les mécanismes d'approvisionnement}

Selon tous les responsables et prestataires interrogés, l'approvisionnement en kits AMIU représente un enjeu particulier dans la perspective de généralisation et surtout de pérennisation des SAA. En effet, jusqu'à l'heure actuelle, l'essentiel des seringues d'AMIU est fourni par les institutions partenaires des pays. Généralement, les premiers approvisionnements sont faits à travers les activités de formation des prestataires. A l'issue de chaque atelier, un kit d'AMIU, comprenant une seringue complète et des canules de tailles diverses, est remis à chaque prestataire formé. Les structures de santé ont profité de ce premier apport pour démarrer leurs activités de SAA, et ont poursuivi l'offre de services avec le même matériel. Certains pays ont pu ainsi offrir des SAA pendant plusieurs années, dans les structures où le programme a été mis en place. Ce fut le cas au Burkina Faso, au Sénégal et en Guinée. Il faut, cependant, remarquer qu'au Mali c'est le Ministère de la Santé qui a acheté les premières commandes de seringues AMIU qui ont été remises aux structures dont leurs personnels ont été formés. Selon un responsable de structure sanitaire et formateur en SAA : "...c'est le niveau national qui a acheté sur commande. $C$ 'est sur commande qu'ils ont payé un stock assez important, parce qu'à ma connaissance, même ce qui doit aller dans les régions ils les ont dans les magasins." Ceci, selon cet interlocuteur, en attendant que, plus tard, les structures sanitaires et les collectivités locales prennent le relais.

Le problème de l'approvisionnement en kits AMIU est survenu avec les premières demandes de renouvellement. En effet, durant les premières années d'introduction des SAA en Afrique francophone, des problèmes se sont posés, car les premières seringues ont vite atteint leur limite 
d'utilisation en termes de nombre d'aspirations (on ne pouvait pas dépasser 100 aspirations avec une seringue), alors que les organismes partenaires ne prévoyaient pas réellement de poursuivre la dotation en matériels d'AMIU au-delà de la formation, et les pays n'avaient pas encore intégré l'idée de mettre en place des systèmes d'approvisionnement en produits SAA. Du reste, le principal souci des responsables gouvernementaux était d'éviter tout risque de dérive avec la mise en place d'un système d'approvisionnement. Ils ont alors toujours émis le vœu d'avoir la maîtrise sur toute la chaîne de commande et de distribution de ce matériel perçu comme étant très sensible. Mais les solutions ont tardé à se concrétiser.

Devant cette situation qui allait aboutir à une impasse, les différentes parties prenantes ont pris des décisions transitoires pour assurer l'approvisionnement des structures chez lesquelles la demande de soins n'a cessé d'augmenter. Les approches utilisées ont différé selon le pays et selon la structure. Si dans certains pays l'approvisionnement est centralisé, comme par exemple au Sénégal, par contre dans d'autres, il est plutôt laissé à la libre appréciation des responsables des structures de santé. Cependant, de l'avis général des personnes interviewées, aucun pays ne dispose encore d'un véritable système d'approvisionnement en matériels d'AMIU, qui impliquerait, entre autres critères, l'introduction dans les normes et protocoles des caractéristiques techniques types des seringues AMIU qui devront être utilisés. De toutes les manières, les organismes partenaires ont dû continuer à fournir du matériel et, à l'heure actuelle, ils assurent la plus grande partie des fournitures d'équipements.

L'absence d'un système d'approvisionnement efficace et pérenne entraîne fatalement des interruptions plus ou moins longues de l'offre de services et favorise la multiplicité des sources d'approvisionnement et donc, par conséquent, une grande diversité de matériels d'AMIU. Au Burkina Faso, par exemple, le système d'approvisionnement n'est pas encore standardisé. Les sources d'approvisionnements en kits AMIU ne sont pas les mêmes. Elles dépendent des partenaires. Des organisations telles que l'OMS et l'UNFPA s'approvisionnement à partir de l'étranger, par le biais de leurs sièges respectifs, tandis que l'ONG Family Care International (FCI) s'approvisionne auprès de IPAS qui a installé un représentant dans le pays. L'OMS a démarré ses actions d'approvisionnement en kits AMIU en 2006. Elle compte, ainsi, renforcer son programme de fourniture de kits de soins obstétricaux d'urgence, qu'elle mène avec l'Union Européenne dans 9 des 13 régions que compte le pays. Elle dispose aussi de son propre fournisseur. Des structures sanitaires cherchent de leur côté à s'approvisionner par le biais d'un autre fournisseur local. C'est le cas du CMA du Secteur 30 à Ouagadougou, dont le médecin chef s'est approvisionné une première fois auprès de GEFCOM, un fournisseur de produits et matériels médicaux établi dans la capitale.

Au Niger, depuis l'introduction des SAA, le pays a bénéficié d'un important appui de FCI pour assurer ses approvisionnements en matériels AMIU. Les demandes étaient exprimées par la maternité Issaka Gazobi qui les transmettait directement à cette institution. Dans les régions où FCI intervient, les seringues sont acheminées directement par ses soins. Ce matériel est acheté auprès d'IPAS depuis son siège à New York. A côté de cette institution, l'UNFPA apporte un important appui en matériel AMIU dans deux régions.

Etant donné les difficultés d'approvisionnement que rencontrent les structures, des solutions sont entrain d'être recherchées dans les pays frontaliers. En attendant, ce sont les membres du personnel qui profitent de déplacements dans les pays de la sous-région pour acheter quelques kits, et se font rembourser par l'administration centrale de leur structure. 
La diversité des sources d'approvisionnement a entraîné de nombreuses différences entre les produits. De ce fait, il se pose souvent des problèmes liés à la méconnaissance des modes d'utilisation qui réduisent considérablement la durée de vie des seringues. De plus, certains produits ne correspondent certainement pas aux conditions économiques des systèmes de santé des pays africains. Par exemple, le CHU Yalgado a reçu une fois des seringues AMIU à usage unique, d'après un des responsables de la maternité de cette structure sanitaire.

Pour juguler ces problèmes, l'un des répondants suggère une centralisation des commandes au niveau de la pharmacie nationale pour avoir des produits uniformes d'une part, et un système d'approvisionnement pérenne sous la tutelle du ministère de la santé, d'autre part. Celui-ci devra alors effectuer des supervisions régulières afin d'assurer un maintien de la qualité des services à un niveau convenable.

Au Niger, c'est FCI qui a assuré le ravitaillement pendant un certain nombre d'années après le démarrage des activités de SAA. Cependant, de l'avis de certains prestataires, l'appui que cette institution apportait à leur institution est entrain de se réduire. De telle sorte que la seringue qui était en service dans l'unité de SAA de la maternité I. Gazobi au moment de l'interview, avait été achetée par une des sages-femmes, lors d'un passage à Dakar. Par la suite, elle s'est fait rembourser par la structure. Pour l'instant, la solution envisagée consiste à envoyer quelqu'un acheter des kits AMIU dans l'un des pays limitrophes.

Dans les pays qui cherchent à centraliser le système d'approvisionnement en matériels AMIU, c'est la structure ministérielle chargée du programme SAA qui rassemble les demandes en provenance des structures sanitaires. Celle-ci adresse des requêtes aux partenaires et organise en retour la redistribution du matériel obtenu. Il arrive dans certains pays que le ministère dispose d'un budget propre pour l'achat de matériels médicaux, dans lequel il peut inclure les kits AMIU. Néanmoins, le fait que les demandes soient adressées à divers partenaires qui achètent les produits chez leurs fournisseurs propres et les livrent au ministère, pose le même problème de la multiplicité des sources et de la diversité des types de seringues et de canules.

Au Sénégal, c'est le centre de santé Roi Baudouin qui achète et stocke les kits pour AMIU depuis juin 2006. Il s'agit d'un arrangement ponctuel initié en l'absence d'un système formel. Il a été facilité par le choix de cette structure par IPAS et par la présence sur les lieux d'un promoteur des SAA, en la personne du médecin chef qui est un formateur en SAA. Cependant, ce système a suscité une incompréhension et des réactions négatives de la part des autres responsables de centres de santé. Le CS Roi Baudouin ayant le monopole de l'approvisionnement et des ventes d'équipements pour $\mathrm{AMIU}$, génère à leur avis des revenus.

Par ailleurs, le processus actuel de commande d'équipements AMIU est lourd et comprend plusieurs étapes : il faut nécessairement faire signer les bons par le ministère de la santé et régler le montant avant de se rendre au centre de santé Roi Baudouin pour s'approvisionner. Les responsables de programme et administrateurs des autres centres de santé estiment qu'une plus grande transparence dans le processus d'approvisionnement en équipements AMIU est nécessaire. Spécifiquement, ils en savent peu sur l'origine et l'identité du fabriquant d'équipements pour AMIU et sur le prix en gros. Actuellement, le prix d'un kit AMIU varie entre 25.000 et 28.000 CFA (environ \$50 à \$55), après vérification auprès du fournisseur. Il semble qu'il faille procéder à un exercice de communication entre les différentes parties prenantes, afin de dissiper les malentendus qui risquent de bloquer le système. 
L'assistance fournie par les partenaires pour l'approvisionnement ne saurait être pérenne, dans la mesure où, selon un des répondants, les partenaires ne pourront certainement pas continuer à appuyer les ministères sans fin. Selon un interlocuteur au Burkina Faso "...tant que on n'est pas arrivé quand même à $50 \%$ d'autonomie par rapport à quelque chose, moi je pense que la pérennité n'est pas garantie..." Il faut donc, selon un autre répondant, intégrer les matériels de SAA dans le plan de sécurisation des produits de SR.

\section{Les solutions qui marchent}

Des solutions ont été mises en œuvre pour assurer l'approvisionnement en kits AMIU, mais elles relèvent essentiellement de structures périphériques. Celles-ci ont développé des mécanismes de recouvrement des coûts et de répartition des ressources qui leur permettent de renouveler leurs équipements en SAA. Il s'agit du CMA du Secteur 30 à Ouagadougou, du CS Roi Baudouin de Dakar et du CMC La Minière de Conakry. Au CMA du Secteur 30, c'est le médecin chef de la maternité qui s'approvisionne directement chez un particulier. Ayant lui-même créé l'unité de soins après avortement dans la structure, il a assuré un premier approvisionnement lors du congrès de la SAGO de Bamako (janvier 2003). Par la suite, un fournisseur local spécialisé dans la vente de matériel médical du nom de GEFCOM lui permet de doter l'unité de soins après avortement en kits AMIU. La source d'approvisionnement n'est pas connue de tous les prestataires interrogés à ce niveau; l'une des sages-femmes sait seulement que cela se fait par le biais du médecin chef qui achète chez un particulier. Au CS Roi Baudouin de Dakar, c'est le Comité de Santé de ladite structure qui fournit les ressources financières et gère le stock de produits par le biais du gestionnaire. L'approvisionnement se fait directement auprès d'IPAS. D'autres centres de santé, à travers le Sénégal, s'approvisionnent auprès du centre Roi Baudouin, grâce aux ressources générées par leurs activités. Enfin, le CMC La Minière de Conakry a mis en place un système de recouvrement des coûts. Et elle assure son approvisionnement en consacrant $35 \%$ de ses recettes mensuelles à l'achat de médicaments et matériels de traitement, dont les kits SAA.

\section{Les solutions envisagées}

Face aux besoins de plus en plus importants des structures offrant des SAA, mais aussi face au défi qu'ont tous les pays de généraliser la pratique sur l'étendue de leur territoire, les dirigeants des Ministères sont entrain d'imaginer des solutions efficaces et pérennes. D'ailleurs, la plupart des répondants pensent comme celui-ci : "...moi je pense qu'il faudrait que nous arrivions à intégrer ça dans nos achats, comme par exemple les médicaments que nous achetons sur le budget national.... Et qu'en fait, les partenaires ne viennent qu'en appui, mais pas comme l'élément, l'assise pour la mise en cuvre de ces programmes." (Un responsable de la DSR, Burkina Faso)

Cet effort est quelques fois accompagné et appuyé par les organismes partenaires, mais pas toujours. Le travail effectué par FCI au Niger est un exemple de démarche qui associe le conseil et l'incitation pour amener le Ministère de la Santé et les structures à développer un système d'approvisionnement qui puissent leur assurer une autonomie. Comme mentionné plus haut, cette institution a soutenu la politique des SAA pendant plusieurs années au Niger. Depuis un certain temps, celle-ci tente de pousser l'administration sanitaire nigérienne chargée de la SR et plus particulièrement les maternités, à développer des initiatives tendant à les rendre autonomes. Elle procède par une sensibilisation et des conseils sur les voies et moyens à utiliser pour réaliser leur autonomie dans ce domaine, accompagnés d'une réduction de ses commandes pour les 
approvisionnements de routine. Ces commandes vont cesser à terme. Par contre, dans une autre institution partenaire basée au Burkina Faso, la solution semble résider dans la poursuite de l'appui pour l'approvisionnement de routine, bien que la nécessité d'organiser un système pérenne eût été exprimée. Le chargé du programme de SR de cette institution a déclaré : "Théoriquement l'achat de kits AMIU est lié à nos programmes, mais je ne vois pas comment on pourra arrêter, tant que nous sommes au Burkina et tant qu'il y aura des avortements."

En Guinée, l'équipement AMIU est maintenant inscrit dans la liste des matériels essentiels, parce que les SAA font partie du paquet minimum d'activité (PMA), étant donné qu'ils ont été intégrés comme élément des soins obstétricaux d'urgence de base (SOUB). "Donc cela a été introduit dans le plan de sécurisation des produits SR au niveau de la direction nationale des pharmacies et laboratoires." (Universitaire formateur, Guinée). Cependant, cette décision n'a pas encore connu un début d'exécution, selon un des répondants.

Dans les autres pays, les équipements pour AMIU ne figurent pas actuellement dans le système national d'approvisionnement, de fournitures et de logistique. Au Sénégal, des discussions sont en cours entre les diverses unités au sein du Ministère de la Santé (DSR, Pharmacie Nationale d'Approvisionnement - PNA) et les partenaires au développement en vue d'inclure les équipements pour AMIU dans la liste des médicaments essentiels. La PNA se chargerait du stockage, et de l'approvisionnement des régions et districts, selon les besoins.

\section{Offres de services en soins après avortement}

Dans le cadre de cette étude, plusieurs types de structures ont été visitées : hôpitaux, centres de santé, structures de référence nationale, structures périphériques équivalant à des centres de santé, postes de santé. L'analyse de l'offre de services dans ces structures a concerné uniquement les maternités et les unités connexes telles que les centres de planification familiale. Dans chaque structure, les équipes de recherche ont visité les unités de SAA, de même que les cliniques de planification familiale, si celles-ci sont séparées des premières citées.

\section{Organisation et continuité des services}

L'ensemble des structures visitées (CHU, CMA, Hôpitaux Régionaux, Postes de santé et Centres de santé) notamment au Burkina, en Guinée, au Mali et au Togo assure des services SAA en continu. Les unités de SAA sont ouvertes du lundi au vendredi. Après les heures ouvrables, les équipes de garde disposent dans certaines structures, d'au moins un membre formé en SAA pour assurer la continuité des services, comme à la maternité I. Gazobi de Niamey.

Par exemple, le personnel de la salle SAA de la maternité Issaka Gazobi de Niamey fonctionne du lundi au jeudi de $8 \mathrm{~h}$ à $16 \mathrm{~h} 30$ et le vendredi de $8 \mathrm{~h}$ à $13 \mathrm{~h}$ en raison de la prière pour les musulmans qui sont majoritaires dans le pays. Mais parfois, il arrive que les prestataires travaillent jusqu'au-delà de $18 \mathrm{~h}$, selon le degré d'affluence des clientes. En dehors des heures d'ouverture de la salle SAA, les patientes sont prises en charge au niveau de la salle d'accouchement. Dans ces conditions, les complications d'avortement incomplet sont souvent prises en charge selon d'autres procédés tels que la D\&C ou le curage digital par les personnels de garde. 
Au Sénégal, l'équipe de recherche a relevé que les services SAA de nuit ne sont pas toujours disponibles dans certaines structures de santé où les patientes sont traitées soit par curage digital, soit renvoyées au lendemain pour des soins par AMIU.

$\mathrm{Au}$ Mali, selon un prestataire, les patientes reçues par les équipes de garde sont envoyées vers d'autres structures : "En dehors de ces horaires, la patiente doit être évacuée vers la structure de référence de Bamako. " (Prestataire Mali). Ceci est dû à l'absence de prestataires formés au sein des équipes de garde.

En Guinée, dans les différentes structures visitées, des dispositions sont prises afin que les soins soient offerts à tout moment. Mais certains des répondants estiment que le matériel n'est pas disponible $24 \mathrm{H} / 24$ au centre de santé de la Minière parce tout le personnel qui assure les gardes n’est pas formé. "La salle est fermée après les horaires normaux de travail. Ainsi, si les patientes SAA ne saignent pas beaucoup, elles attendent le lendemain pour être traitées". (Prestataire Guinée).

Une autre raison est liée à l'indisponibilité, pendant la nuit, du matériel pour AMIU qui est parfois mis sous clé ; et seule la personne responsable des SAA y a accès. Certains prestataires justifient cela en précisant que la garde des équipements était une précaution pour empêcher l'utilisation de ce matériel ${ }^{8}$ à d'autres fins. Dans une structure de référence de Bamako, la solution suivante est utilisée afin d'assurer la continuité des SAA, tout en assurant la sécurité de l'emploi du matériel : "...en ce moment au niveau du service gynéco, qui en même temps notre service à nous, à la descente le matériel est amené au niveau du bloc opératoire pour l'AMIU pour éviter que ce soit très clandestin, quand quelqu'un vient ouvrir la salle gynéco seul parce que c'est très distant. Il faut que ce soit par équipe de garde au vu et au su de tout le monde, [et] enregistré." (Responsable de maternité, Bamako). Au Togo, dans un centre périphérique, la détérioration du matériel AMIU, devenu inutilisable, et la dislocation de l'équipe SAA a interrompu la fourniture des services depuis plusieurs mois.

La non disponibilité du matériel d'AMIU pendant les heures de garde favorise la survivance de la pratique du curage digital et de la D\&C qui, souvent, ne s'accompagnent pas de counseling et entraînent quelques fois des douleurs pour les patientes.

Par ailleurs, les personnels ont mis en exergue les problèmes qui se produisent avec un afflux important de patientes. Dans ces situations, le nombre de seringues (généralement pas plus de deux par structure) et le temps nécessaire à leur désinfection ou leur stérilisation, font que les prestataires souvent sont obligés de faire recours aux autres méthodes d'évacuation utérine. "En cas de grande affluence, certains prestataires sont obligés d'utiliser d'autres méthodes d'évacuation utérine face à l'urgence, en attendant que le matériel soit en condition". (Prestataire Burkina).

\footnotetext{
${ }^{8}$ L'équipe n'a pas cherché à découvrir si l'utilisation à d'autres fins des équipements pour AMIU était une réalité.
} 


\section{Prestations offertes en SAA}

Lors de la Conférence de 2002 sur les SAA, un modèle à 5 composantes a été introduit en remplacement de celui à 3 composantes qui était en vigueur lors des premières expérimentations. Les 5 composantes essentielles sont les suivantes :

- Le traitement de l'avortement incomplet;

- Le counseling;

- L'offre de services contraceptifs et de PF ;

- Le lien avec d'autres services ;

- Le partenariat avec la communauté.

L'analyse des prestations offertes en SAA sera faite selon ces 5 composantes.

\section{Le traitement de l'avortement incomplet}

L'essentiel des complications d'avortements reçues dans les structures visitées a été traité par AMIU. Ce matériel a permis d'alléger considérablement les conditions de prise en charge des patientes, grâce à sa facilité d'utilisation et de conditionnement. En effet, avec l'AMIU, il a été possible d'impliquer et de responsabiliser pleinement les sages-femmes et quelques fois les infirmiers dans la prise en charge des urgences. Cette catégorie de prestataires étant plus nombreuse dans les structures sanitaires, elle a permis de raccourcir les délais entre l'admission et la prise en charge effective. Dans toutes les structures visitées, l'autonomisation des locaux réservés aux SAA, a permis un accès direct des patientes aux soins et leur a ainsi évité d'être mises en concurrence avec d'autres types de complications gynécologiques ou obstétricales qui sont souvent considérées comme prioritaires.

Cependant, dans certaines structures, il n'existe pas encore de local réservé aux SAA à cause de contraintes logistiques qui perdurent. C'est le cas dans une structure sanitaire à Bamako où le traitement des complications d'avortement est effectué au niveau du bloc opératoire lorsqu'il est disponible, ou bien dans la salle de consultation des urgences de la maternité. Une telle situation pose à priori un problème de gestion efficace du matériel et de continuité de tout le paquet de services du modèle de SAA. En effet, le rythme d'utilisation d'un bloc opératoire n'offre pas le temps nécessaire pour mener un bon counseling pré, per et surtout post AMIU.

La salle de consultation des urgences est généralement un local plus ou moins grand avec plusieurs lits. Elle n'offre pas la confidentialité requise dans le cas de prise en charge des questions d'avortement.

L'on retrouve d'ailleurs cette situation dans toutes les structures visitées, notamment pendant les gardes où l'unité de SAA est fermée. Dans ces cas, les complications d'avortement sont traitées dans les salles d'accouchement ou bien dans les blocs opératoires. Cette solution semble acceptée par tous les prestataires interrogés. Mais, la question est de savoir si cette solution contribue efficacement à la qualité des SAA. Cette situation pose aussi la question de la raison pour laquelle il n'est pas possible d'accéder à la salle de SAA, pendant les heures de garde, dans la mesure où celle-ci dispose généralement de tous les éléments nécessaires, aussi bien pour le traitement que pour le counseling en PF.

\section{Le counseling}

C'est la composante qui intervient à toutes les étapes de la prise en charge des complications liées aux avortements incomplets. En effet, de l'accueil à la référence éventuelle de la patiente, en passant par le traitement et l'offre de méthodes contraceptives, le modèle de SAA préconise le 
dialogue permanent entre le personnel traitant et la patiente. Ceci, afin de la mettre en confiance, de comprendre ses problèmes et ses besoins, et de l'informer sur la conduite à tenir devant certaines situations. Le terme "dialogue" implique des échanges oraux entre la patiente et le prestataire et qui sont spécifiques à chaque étape de la prise en charge.

En général, les prestataires ont affirmé faire le counseling aux patientes. Mais il s'avère qu'il n'y a pas réellement d'échanges, surtout au moment du traitement. Ce sont plutôt des directives qui sont données par le personnel, ou bien à la limite des questions qui attendent des réponses du type OUI/NON, qui sont posées.

Le seul moment où l'on peut penser qu'il y a counseling, est celui pendant lequel le personnel aborde la question de la contraception. Les prestataires interviewés dans cette étude ont parlé du counseling, mais en mettant l'accent sur l'offre de services de planification familiale. En effet, la plupart des prestataires se sont exprimés longuement sur leur conviction quant à l'importance du counseling après les SAA et de l'utilisation de méthodes contraceptives après un avortement. La vérification de l'effectivité du counseling est généralement faite lors des "visites de retour", qui ont lieu une semaine après le traitement d'urgence. Néanmoins, il a été noté que, contrairement au counseling PF après traitement, le counseling avant et pendant le traitement d'urgence n'est pas systématique. L'équipe de recherche a trouvé que les salles SAA visitées disposaient du matériel pour le counseling en PF. Cependant, ce matériel reste toujours dans ces locaux et n'est pas accessible aux équipes de garde.

\section{L'offre de services contraceptifs et de PF}

Dans certaines structures de santé la méthode est offerte immédiatement, par contre dans d'autres, les patientes sont référées à l'unité de PF après le counseling. Cette entité peut-être située dans la structure sanitaire ou en dehors.

En fait les structures des six pays visitées disposent d'une gamme de produits contraceptifs au niveau de l'unité PF, à l'exception parfois du Norplant ${ }^{\circledR}$ et du DIU. Mais il faut noter que ces contraceptifs ne sont pas en général disponibles dans les salles de traitement d'urgence, ou de récupération des femmes ayant bénéficié des SAA, à l'exception de la Guinée où l'équipe a trouvé en salle d'urgence, et ceci au niveau de deux structures visitées, toutes les méthodes contraceptives sauf les implants et le Condom féminin.

Au Sénégal, des contraceptifs oraux étaient disponibles en salle de récupération après SAA au niveau d'un seul centre de santé. Au niveau de ce pays, les raisons évoquées concernant la non disponibilité des produits seraient liées à un problème de gestion des produits SR ; les contraceptifs étant inclus dans la liste des médicaments essentiels et gérés par leurs comités de santé au niveau de leurs dépôts de produits pharmaceutiques.

Au Mali, seuls les contraceptifs oraux étaient disponibles dans les unités de SAA. Dans les cas où les patientes désiraient utiliser d'autres méthodes, elles étaient référées vers l'unité de PF qui était quelques fois située en dehors de la structure.

Ainsi, après le counseling, la femme qui choisit une méthode spécifique est souvent envoyée vers l'unité de PF (qui fonctionne uniquement pendant les jours et heures de travail) où elle reçoit une ordonnance qu'elle doit amener à la pharmacie pour pouvoir acheter sa méthode contraceptive. Il n'y a pas de données disponibles sur le nombre de femmes ayant suivi ce circuit. 
Cette situation observée au Mali, au Burkina, au Sénégal et au Niger fait que certaines femmes peuvent rentrer chez elles après leur prise en charge sans bénéficier de méthode contraceptive. Cependant, au Niger, il arrive que les patientes à qui une méthode a été offerte dans l'unité de SAA de la maternité I. Gazobi, soit suivies par la suite par le même personnel pour la continuité de leur contraception.

\section{Le lien avec d'autres services}

La relation entre les différents services de SR est devenue, de nos jours un moyen pratique pour réaliser l'intégration des services. Cette stratégie vise, entre autres objectifs, à profiter de la présence des patientes dans les structures pour mettre à leur disposition plusieurs services dont elles pourraient avoir besoin. A ce titre, les SAA, dans leur définition, contribuent à la réalisation de cette stratégie en intégrant l'offre de méthodes contraceptives aux patientes et en organisant la continuation de la pratique par une référence vers les unités ou centres de PF les plus accessibles. Toutefois, dans la pratique, la référence des patientes à qui une méthode a été remise, lors du traitement de l'avortement, vers les unités de PF, en vue de la continuation de la contraception, n'est pas bien documentée. De ce fait, il est difficile de tirer des conclusions sur son effectivité, d'autant plus que dans tous les sites visités, l'unité de SAA est distante de celle de PF et, surtout, il n'existe pas de liens formels entre ces deux entités. La remontée des informations se fait généralement à partir d'une initiative individuelle et n'offre pas de garantie quant à leur complétude.

L'équipe de recherche a remarqué la concrétisation d'un autre aspect de la relation avec d'autres services en Guinée et au Niger, où les patientes de plus de 24 ans sont systématiquement référées vers une structure située en dehors pour la détection du cancer du col de l'utérus. L'effectivité de la référence est vérifiée lors de la visite de contrôle, qui a lieu une semaine après le traitement.

Cette initiative ne semble pas être systématisée dans les structures sanitaires visitées lors de l'enquête.

Il y a lieu, cependant, d'optimiser la référence pour le dépistage de cancer du col de l'utérus, dans la mesure où, l'incidence de cette maladie serait assez faible au plan mondial ( 23 pour 100000 femmes). L'idée serait de profiter du moment que les prestataires consacrent à l'anesthésie para cervicale, pour procéder à une inspection visuelle (au lugol -IVL- ou à l'acide acétique -IVA). Cela permettrait de faire un premier tri à moindre coût et, ainsi, de ne référer que les cas suspects. Le dépistage du cancer du col de l'utérus par IVL ou par IVA se fait déjà par les sagesfemmes. De plus, à priori, l'introduction de cette activité ne devrait pas apporter un surplus de travail important pour les prestataires, et ne devrait pas prolonger de manière significative la durée de la prise en charge.

Au Burkina Faso, les services les plus sollicités pour la référence sont la gynécologie, les services d'IST, de dépistage du cancer du col et à des degrés moindres le service d'urologie, de santé mentale, mais également les centres de jeunes (centres de conseils ados) pour les adolescentes.

Par ailleurs, habituellement les autres services sollicités sont la cardiologie, la médecine interne pour la prise en charge des diabétiques ou des personnes vivant avec le VIH. 
Les données montrent que le système de référence/contre référence devrait être amélioré et renforcé. L'information sur l'endroit où la patiente a été référée et sur son nouveau statut n'est pas toujours disponible.

Il est à noter que, dans aucun des pays, l'étude n'a montré le lien entre l'avortement et le programme de lutte contre le paludisme. Ceci, sachant que cette maladie constitue, en fait, une des premières causes d'avortement. Ce lien pourrait se concrétiser par un appui dans le dépistage du parasite, le traitement curatif si nécessaire et la prévention par la fourniture de moustiquaires imprégnées.

D'après les répondants, les conditions d'évacuation d'une patiente diffèrent selon que l'on se trouve au niveau du CHU (là il s'agit juste de l'orienter vers le service approprié selon le cas), du CS, du PS ou du CMA. Il n'a pas été noté de registre spécifique pour les évacuations ou références des patientes SAA aussi bien au Niger, au Mali, au Burkina, en Guinée qu'au Sénégal. Quand elles existent, seules sont établies des fiches de référence ou fiches de liaison selon les pays.

\section{SAA et communauté}

\section{Le partenariat avec la communauté}

En fait, l'introduction de cette composante a entraîné des changements dans la logique qui soustend le modèle de prise en charge des complications d'avortement. Le modèle à trois composantes était surtout centré sur la patiente à l'intérieur de la structure. Et, logiquement, la prise en charge devrait démarrer avec le traitement de l'urgence et finir avec l'offre de méthodes contraceptives et les conseils sur la conduite à tenir en cas de besoin. Le tout se déroulant au sein des structures de santé.

Le partenariat avec la communauté fait démarrer la prise en charge dans l'environnement immédiat de la patiente. Et, dans ce contexte, les membres de la communauté doivent être capables de détecter les signes d'un avortement en cours, de prendre les premières mesures d'urgence et d'organiser la référence vers la structure la plus indiquée. Plusieurs études ou expériences menées en Afrique et ailleurs ont montré la viabilité et la pertinence d'une telle approche. Le rapport analytique de la Conférence sur les SAA en 2002 proposait un modèle de partenariat avec les communautés, qui figure dans l'encadré suivant.

\section{Encadré 2 : Principales méthodes d'engagement communautaire dans la gestion des complications de grossesse et des soins après avortement}

- Identifier un représentant à partir de la communauté.

- Donner à la communauté des informations sur les avantages de l'utilisation des services.

- Former les matrones et autres agents de santé communautaires ou villageois.

- Accroître les connaissances de la communauté sur les signaux d'alerte à travers les matrones et autres agents.

- Établir un système de transport d'urgence comportant :

- un plan d'évacuation ;

- un fonds pour les urgences et remboursements ;

- un moyen de transport ;

- un moyen d'évacuation ;

- un système pour surveiller les femmes qui ont été évacuées. 
Cependant, en dehors de quelques institutions comme FCI au Burkina Faso et au Niger, peu d'acteurs intègrent réellement cette composante dans leur programme. D'ailleurs, la démarche d'introduction des SAA dans les pays est restée inchangée, avec une entrée en matière dans les structures de niveau national, puis dans les structures de référence régionale et de district, pour arriver au niveau primaire et finir par le niveau communautaire. Ce qui fait que plus de dix ans après l'introduction des SAA en Afrique francophone, peu de pays n'ayant pas pu franchir toutes les étapes, par conséquent peu d'actions ont été faites pour encourager les populations à voir l'avortement comme un problème de santé publique qui doit être adressé avec toutes les ressources nécessaires.

Généralement, les relations que les structures ont avec les communautés se limitent à l'information et la sensibilisation sur l'existence de possibilité d'être traitées avec une nouvelle méthode moins douloureuse et moins risquée que celles qu'elles connaissent. Peu ou aucune action d'éducation n'est menée en direction de ces communautés.

L'action de FCI au Burkina Faso et au Niger se résume en trois grandes étapes décrites comme suit :

- Dans une premier temps, les agents du premier niveau de la pyramide sanitaire ont été formés pour maîtriser les gestes de base : "...il y a eu des compétences qu'on a donné aux prestataires de premiers niveaux qui peuvent leur permettre d'abord d'identifier, de diagnostiquer les complications qui peuvent être liées aux problèmes d'avortement et de les référer en cas de nécessité. Aussi les autres aspects qui peuvent permettre déjà de stabiliser l'état d'une femme qui vient avec un problème d'avortement avant de la référer." ;

- Ensuite, les relations avec les communautés leur ont été enseignées "Ils ont aussi appris le lien à établir avec la communauté pour que d'une manière ou d'une autre la sensibilisation se fasse pour que bon déjà à ce stade là, les gens puissent prendre des dispositions soit au niveau communautaire, soit dans le cadre du transfert de malade pour pouvoir faciliter l'accès aux soins." Pour ce faire, un matériel didactique adapté à la cible a été utilisé. Il s'est agit de pictogrammes et de boîtes à images qui ont été testées dans d'autres pays, et dont le contenu a été modifié en fonction du contexte local ;

- Et enfin, "...nous avons mis en place comme éléments de soins l'AMIU, l'aspiration manuelle intra utérine qui était vraiment l'aspect novateur dans la prise en charge des femmes avec complications obstétricales".

L'application de ce modèle à cinq composantes nécessite néanmoins la réalisation de plusieurs conditions au préalable. L'une des conditions les plus importantes est la mise en place d'une politique nationale qui définisse et codifie les règles de fonctionnement des structures à tous les niveaux de la pyramide sanitaire, y compris les liens et horizontaux et verticaux qui doivent régir les relations entre elles. En plus, cette politique devrait définir clairement le rôle des communautés, en insistant sur les moyens à mettre en œuvre pour les responsabiliser et les aider à s'organiser en conséquence. Ces mesures devraient être accompagnées d'un cadre juridique et législatif, qui mette en place les instruments garantissant l'accès à des services de qualité, grâce à une gestion équitable et efficace des ressources (humaines, matérielles et financières). Les problèmes soulevés précédemment montrent l'ampleur des efforts à fournir pour parvenir à offre des services après avortement en respectant tous leurs aspects.

Dans tous les pays visités, les aspects médicaux ont été mis en avant en oubliant le cadre réglementaire qui devrait définir les rôles des différents acteurs, de même que les moyens et 
ressources qui devraient permettre leur mise en œuvre. Finalement, à cause de ces manquements, continuent d'être la responsabilité des bailleurs et se limite à l'offre de quelques services dans les structures sanitaires. C'est ce que résume un responsable de maternité à Bamako : "Le modèle enseigné c'est un modèle normalement à cinq composantes, mais le modèle qui est le plus souvent exécuté sur le terrain c'est le modèle à trois composantes. Souvent ce sont les contraintes objectives du milieu qui l'imposent".

\section{Volume d'activité}

De manière générale, le niveau d'appréciation du volume d'activités dans les pays ciblés diffère en fonction de la structure, du nombre de prestataires impliqués et de leur degré de responsabilité dans l'unité SAA.

Les statistiques n'ont pas pu être observées dans toutes les structures, mais les registres consultés font apparaître que l'essentiel des activités SAA sont menées par les sages femmes formées. Dans ces pays, l'implication des médecins dans la prise en charge des cas d'avortement est souvent liée aux cas compliqués.

D'après les données disponibles, le nombre de cas varie selon le type de structures. Au Burkina Faso, pour le CHU Yalgado, la sage-femme responsable de l'unité SAA et sa collègue affectée dans le service ont pris en charge respectivement au cours du mois de mars 2007, 40 et 37 femmes pour des soins après avortement. Durant le premier trimestre de la même année, le nombre de clientes tournait autour de 98 et 110 femmes. Leur présence quotidienne dans l'unité de SAA aux heures de travail explique le nombre important de femmes prises en charge pour des complications liées à un avortement incomplet. Ces résultats ne sont pas comparables à ceux des médecins interrogés dans la même structure. Leur implication pour la prise en charge des cas d'avortement est souvent sujette à leur tour de garde ou à des cas de complications non gérées par les sages-femmes. Le nombre de cas gérés ne dépasse pas quinze (15) par mois, y compris ceux traités par ces médecins dans les structures privées.

Au niveau du CMA du Secteur 30, la situation est différente, seules les sages-femmes ont répondu à cette question. La responsable de l'unité SAA reconnaît avoir traité 8 cas au cours du mois de mars et seulement 16 pendant le premier trimestre 2007, compte tenu de son absence d'un mois pour raison de congé. Sa collègue et adjointe a reçu et pris en charge 30 femmes pour des complications liées à un avortement incomplet durant le mois de mars et 67 pendant le trimestre.

L'ensemble des cas reçus par les prestataires interviewés est correctement pris en charge par ces derniers à l'exception de cinq selon l'une des sages-femmes du CHU. La raison évoquée pour cette défaillance est le degré de complication de ces cas lié à des IVG par l'ingurgitation de produits abortifs non indiqués d'où la nécessité d'une orientation pour une meilleure prise en charge.

Etude de cas du Sénégal : ce tableau montre le nombre de cas au niveau de 7 centres et 2 hôpitaux ; de janvier à juin 2006. 
Tableau 3 : Statistiques sur les activités de SAA dans trois types de structures sanitaires au Sénégal, de janvier à juin 2006

\begin{tabular}{llll}
\hline \hline & $\begin{array}{l}\text { CS I } \\
\text { (Nombre = 5) }\end{array}$ & $\begin{array}{l}\text { CS II } \\
\text { (Nombre = 2) }\end{array}$ & $\begin{array}{l}\text { Hôpital } \\
\text { (Nombre = 2) }\end{array}$ \\
\hline Nombre d'accouchements & 2944 & 1077 & 662 \\
Nombre de patientes de SAA & 292 (fourchette 6-100) & 213 (fourchette $70-94)$ & 191 \\
Nombre de patientes traitées par AMIU & $206(71 \%)$ & $147(69 \%)$ & $98(51 \%)$ \\
Patientes traitées par curage digital & $67(23 \%)$ & $59(28 \%)$ & $60(28 \%)$ \\
Patientes traitées par D\&C & 0 & $7(3 \%)$ & $25(12 \%)$ \\
Patientes référées & 81 & 69 & 0 \\
Patientes conseillées en PF & $285(95 \%)$ & $185(87 \%)$ & $165(86 \%)$ \\
Patientes ayant reçu une méthode : & 173 & 48 & 85 \\
$\%$ ayant reçu une méthode & $59 \%$ & $29 \%$ & $45 \%$ \\
$\%$ des patientes conseillées & $61 \%$ & $34 \%$ & $52 \%$ \\
\hline \hline
\end{tabular}

NB : Les centres de santé (CS) dispensent des soins de santé primaire, des services obstétricaux et d'autres services de SR, ils disposent par ailleurs de structures d'hospitalisation de courte durée. Ils sont de deux types selon qu'ils comportent ou non une salle d'opération : le Type II (CS II) possède une salle d'opération et le Type I (CS I) n'en possède pas. Le personnel comprend des gynécologues, des généralistes, un chirurgien et un anesthésiste (dans les CS disposant d'une salle d'opération), des sages-femmes, des matrones traditionnelles, des infirmières et des aides infirmières.

En Guinée, au CHU, le médecin responsable de l'unité de soins après avortement n'a pas reçu de cas au cours du mois qui a précédé la venue des enquêteurs. Cela est dû au fait que le responsable de l'unité est beaucoup plus occupé par la formation en SAA, dans le cadre de l'extension. Cependant il reconnaît avoir reçu 5 cas au cours du dernier trimestre. Par contre l'adjoint au responsable SAA affirme avoir traité au moins 3 cas sans donner de précision pour le trimestre, tandis que la surveillante générale de la maternité évalue le nombre de cas reçu dans la même période à une dizaine avec un cumul d'environ trente cas. Un prestataire reconnaît ne pas avoir pris en charge au cours du mois dernier et n'a pas de souvenir sur le nombre réalisé pendant le dernier trimestre.

Au centre de la Minière, seuls deux prestataires ont apportés des réponses, ils ont reçu chacun trois cas au cours du mois précédent la visite des enquêteurs, et sept et huit cas pendant le dernier trimestre. Les autres prestataires ne savent pas ou ne font pas de services de SAA complets (la chargée de l'unité PF).

Les cas de complications liées à un avortement incomplet évoqués ont tous été pris en charge par les prestataires interrogés à l'exception d'un cas au CHU qui présentait un tableau de complication en péritonite ou pelvipéritonite et qui est du ressort du service d'accueil selon le prestataire.

Au Niger, la maternité Issaka Gazobi a reçu et traité 90 cas d'avortement au mois de mai 2007, que cela fût en salle SAA ou en salle d'accouchement. La responsable de la salle SAA en a assuré le tiers. Les autres ont été pris en charge par l'autre sage-femme du service SAA et par les équipes de garde en salle d'accouchement. 
A Mali, au CHU G. Touré et au CS de la Commune 5, au cours du mois ayant précédé l'enquête, les médecins et sages-femmes enquêtés ont pris personnellement en charge entre 2 et 10 patientes, soit, en moyenne, 4 clientes par prestataire. Au cours des 3 derniers mois, 4 prestataires ont effectué 6 SAA chacun en moyenne. Un prestataire a plutôt supervisé les apprenants et un autre prestataire n'a pas eu à en faire de prestations. Il faut noter que, d'une manière générale dans les 2 sites, les sages-femmes pratiquent plus que les médecins.

\section{Coût des services}

Au niveau du tableau ci-dessous, une description des coûts selon les pays visités :

\section{Tableau 4 : Coûts des services de SAA dans les 6 pays selon le niveau des structures} sanitaires

\begin{tabular}{|c|c|c|}
\hline Pays & Coûts des services & Structures sanitaires \\
\hline Guinée & $\begin{array}{l}\text { - } 15000 \mathrm{FG}^{*} \text { (Acte) + } 5000 \mathrm{FG} \text { pour médicaments) } \\
\text { - } 10000 \mathrm{FG}^{*} \text { (Acte + produits) }\end{array}$ & $\begin{array}{l}\text { CHU Donka } \\
\text { CS La Minière }\end{array}$ \\
\hline Mali & $\begin{array}{l}\text { - Acte gratuit + produits : } 6000 \text { FCFA } \\
\text { - } 7500 \text { FCFA (Acte + produits) : entre } 6000 \text { FCFA et } 7000 \\
\text { FCFA }\end{array}$ & $\begin{array}{l}\text { CHU Bamako } \\
\text { CS de la Commune }\end{array}$ \\
\hline Togo & $\begin{array}{l}\text { - } 7550 \text { FCFA (Acte) + } 6000 \text { FCFA pour médicaments } \\
\text { - } 15000 \text { FCFA (Acte + médicaments, antibiotiques non } \\
\text { compris) }\end{array}$ & $\begin{array}{l}\text { CHU Lomé } \\
\text { Hôpital Bè }\end{array}$ \\
\hline Burkina & - 14000 FCFA (Acte + produits et médicaments) & CHU Yalgado \\
\hline Sénégal & $\begin{array}{l}\text { - } 10000 \text { FCFA (Acte + produits) } \\
\text { - } 7500 \text { FCFA (Acte + produits) }\end{array}$ & $\begin{array}{l}\text { Hôpitaux régionaux } \\
\text { CS }\end{array}$ \\
\hline
\end{tabular}

Le coût de l'ordonnance post soins n'est pas compris dans les coûts ci-dessus / * 7 Francs Guinéens (FG) = 1 FCFA au moment de l'enquête ; 1 \$=440FCFA.

Dans les pays de la zone franc, le coût des services varie de 6000 à 15000 FCFA (\$14-\$34), compte non tenu de l'ordonnance de suites de couches et des produits contraceptifs. Dans un même pays, cette disparité de la tarification doit amener les autorités de tutelle à initier une politique d'harmonisation, de préférence à la baisse, du coût des services. Car, en Guinée, le coût des services des SAA n'excède pas 3000 FCFA environ (7\$).

Les avis recueillis auprès de la communauté révèlent que les coûts des prestations constituent pour certaines patientes un frein pour pouvoir bénéficier des services SAA. Ces barrières financières ont été mentionnées aussi bien par les femmes que par les hommes lors des discussions de groupe.

"Les prix sont trop élevés, il faut les diminuer pour aider les femmes " (Femme/Burkina)

"Je préfère acheter de l'aspirine à la pharmacie ou boire une citronnade ou des pulpes ...c'est moins coûteux..." (Femme / Sénégal)

En plus du prix de la prestation, les femmes doivent souvent acheter un bon nombre de produits comme l'eau de javel, les gants et les analgésiques. Si elles souhaitent commencer une méthode contraceptive, elles doivent prendre en compte le prix du produit. Leurs dépenses augmentent lorsqu'elles doivent en plus payer le transport.

"...nous payons un taxi : 1000 F CFA pendant la journée et 2000 F CFA la nuit ... "...parfois nous vendons une part de nos marchandises pour payer le transport de nos femmes... "

(Homme/Sénégal) 
Par ailleurs, les participants ont signalé que les femmes consultent parfois les matrones traditionnelles par faute de moyens. Ces dernières demandent environ 1000 ou 2000 FCFA pour leurs services, et puisqu'elles résident au sein de la communauté, le problème du transport ne se pose pas.

"...pour atteindre la case de santé, il faut 5 minutes, deux heures pour arriver à l'hôpital..." (Femme/Sénégal)

Les coûts des services et les difficultés financières ont également été soulevés par les membres des comités de santé. Dans certaines communautés, ces comités s'organisent et participent aux frais en offrant des montants assez variables ; certains ont mis en place des stratégies de mobilisation de fonds telle que la vente des moustiquaires. D'autres épargnent des fonds pour le transport en cas d'urgence, en particulier pour les patientes indigentes.

"...un fonds de solidarité est mis en place dans les villages pour le transport d'urgence des femmes enceintes ..." (Homme/Sénégal)

"...souvent, les chefs religieux collectent des fonds pour le transport d'urgence des femmes enceintes ..." (Femme / Sénégal)

Dans l'ensemble, les répondants estiment que les coûts des SAA sont élevés pour la communauté.

"C'est un peu cher, tout le monde n'a pas les moyens, y a des femmes qui peuvent payer, mais y en a d'autres qui n'ont pas vraiment les moyens, elles peuvent rester à la maison par manque de moyens hein, et continuer à saigner ". (Prestataire/ Mali)

\section{SAA et cases de santé : Cas du Sénégal}

Chaque communauté au Sénégal possède une case de santé ; cependant, la majorité de ces cases ne fonctionne pas. Le MSPM fait des efforts pour établir des services minimums dans toutes les cases qui sont une responsabilité de la communauté et sont financées par des fonds communautaires. Deux cases de santé assez accessibles ont été visitées pour jauger leur capacité à participer à l'offre des services de SAA. Dans ces sites, le personnel est composé de sept matrones, dont trois formées en SAA. Ces matrones sont responsables de la prise en charge des accouchements sans complications, des vaccinations et autres services concernant la survie de l'enfant. Elles n'offrent pas de counseling sur le planning familial, même pas celles qui sont sensées avoir reçu une orientation en SAA. L'équipe n'a pas pu déterminer si ces matrones travaillent également comme accoucheuses traditionnelles au sein de la communauté.

Les deux cases visitées sont confortables et propres, avec de l'eau courante, un bon éclairage, des toilettes en bon état, et un endroit pour les accouchements dans l'une d'entre elles. Un minimum d'équipements et de matériels de prévention de l'infection (spéculums à deux valves, forceps, gants stériles, boite à tranchants, eau de javel) et des antalgiques y sont disponibles, mais les méthodes de planning familial, notamment les condoms font défaut. Trente huit (38) femmes ont accouché au niveau de ces deux cases entre janvier et juin 2006 et l'une d'entre elles a été référée. Aucune patiente ayant des complications après avortement n'a été enregistrée, et aucune femme n'a reçu de counseling PF. 
La plupart des prestataires interviewés dans les centres et postes de santé estiment qu'il existe un potentiel pour étendre des activités « ciblées » de SAA aux cases de santé concernant les aspects suivants : l'éducation communautaire sur la prévention de la grossesse et les signes d'alerte pendant la grossesse, l'identification des complications, la référence rapide et l'assistance en moyens de transport dans les cas d'urgence, le suivi des femmes consultées dans les structures sanitaires, le counseling PF et la distribution des méthodes de PF choisies. Aucun enquêté n'a recommandé que les traitements d'urgence soient dispensés dans ces sites.

\section{Perceptions des patientes sur la qualité des services}

La majorité des patientes SAA (Burkina, Mali, Guinée, Niger) sont, dans l'ensemble, satisfaites des services reçus. Les différents prestataires interrogés l'attestent et donnent un certain nombre d'éléments d'appréciation. Il est apprécié le temps d'attente qui se trouve réduit avec la technique AMIU et selon les prestataires, les patientes trouvent l'AMIU moins coûteux compte tenu du paquet de services qui est en même temps offert. La gestion de la douleur également est un point très important soulevé par les patientes. "Ha, vraiment madame, j'avais tellement peur, je croyais que ça faisait mal parce que, la dernière fois, j'ai subi un curetage qui m'a vraiment traumatisée."(Patiente/Mali)

Aussi, l'interaction avec le prestataire par le biais du counseling tout au long de la procédure est bien appréciée par les patientes qui trouvent que l'AMIU est moins traumatisante. Le degré de satisfaction de ces patientes est davantage perçu à travers le retour de certaines patientes dans la structure pour tout problème de santé les concernant. "Leur satisfaction est même visible quand on fait la comparaison entre leur arrivée (inquiétude) et la sortie avec le sourire ou les remerciements ". (Prestataires/Guinée)

\section{Opinions de la communauté sur l'offre des services}

Néanmoins, des barrières liées à la qualité des services ont été notées lors des discussions de groupes dirigées. Certaines femmes ont évoqué le comportement parfois irrespectueux envers elles, ce qui les pousse d'ailleurs à s'adresser à la matrone en cas de problème plutôt qu'à la sage femme.

"...les femmes préfèrent habituellement trouver les matrones chez elles, parce qu'elles sont plus expérimentées et elles sont gentilles..." (Femme/Sénégal)

"...certains prestataires insultent et maltraitent les femmes célibataires venues pour des SAA..." (Femme/ Sénégal)

"...elles sont parfois agressives, et nous insultent..., elles devraient être plus humaines, plus accueillantes et plus gentilles ". (Femme/Burkina)

\section{Perception et attitudes des prestataires vis à vis des femmes et jeunes filles ayant fait des interruptions volontaires de grossesse}

Avec un pourcentage de 5 à $30 \%$ environ selon les prestataires, l'IVG occupe au Burkina, au Niger, en Guinée et au Mali une place non négligeable dans le lot des complications liées à l'avortement incomplet. Ce pourcentage assez élevé trouve son explication dans la persistance de rapports sexuels non protégés.

Les adolescentes victimes de l'IVG sont très souvent en bas âge (entre 15 et 18 ans) et dans une ignorance totale des conséquences. Ce sont des adolescentes souvent illettrées, mais parfois aussi des étudiants. 
Le profil des femmes qui pratiquent les IVG montre que toutes les catégories sont concernées. A quelques exceptions près, on retrouve les mêmes cas de figure dans les pays visités.

Pour les prestataires, la prise en charge de ces cas d'avortement ne diffère pas par rapport aux cas d'avortements spontanés. Le counseling est fonction de la cible selon qu'on soit adolescente, célibataire, veuve ou mariée. 


\section{CONCLUSION ET SUGGESTIONS}

La conférence de 2002 sur les soins après avortement avait été un moment clé dans le développement de l'initiative consistant à promouvoir leur introduction dans les pays d'Afrique francophone. Tous les thèmes relatifs aux SAA ont été abordés. Cependant, étant donné que la plupart des pays participants n'en n'étaient qu'à leurs débuts, les grands défis de l'époque étaient d'identifier les voies et moyens pour introduire les SAA dans les pays. A cet effet, le modèle suivant avait été proposée ${ }^{9}$ :

- un champion local qui demeure activement impliqué depuis la décision d'introduire le nouveau modèle de services jusqu'à l'adoption totale du programme par les parties prenantes appropriées ;

- le plaidoyer pour expliquer la nécessité, la simplicité et les avantages des services de SAA ;

- le respect pour les droits des clientes par les officiels, administrateurs, prestataires et autres ;

- un programme vertical dès le départ pour clairement démontrer les effets du nouveau modèle de services ;

- initialement quelques sites pour garantir une qualité élevée grâce à des efforts concentrés et faciliter la supervision ;

- une phase intensive d'introduction, peut être une étude de recherche opérationnelle ou un projet pilote avec un volet d'évaluation rigoureuse, accompagnée suffisamment par une assistance technique et un soutien financier externes ;

- une formation par des formateurs qualifiés et expérimentés ;

- une surveillance et une supervision intensives pour garantir que les prestataires continuent d'appliquer les nouvelles compétences et identifier toute barrière potentielle au succès de l'intervention;

- équipements et fournitures nécessaires pour tous les services.

A l'évidence, l'idée principale de ce modèle était de mettre un accent particulier sur le plaidoyer et sur une bonne image que les acteurs devaient donner aux SAA, afin d'en faciliter l'acceptation par les décideurs. C'est ainsi que ce modèle décrit un processus que, du reste, le Burkina Faso, le Sénégal et la Guinée avaient suivi pour introduire les SAA.

Certaines propositions de ce modèle ont été rappelées par les répondants à cette présente évaluation, comme par exemple celles relatives au leadership assuré par des champions, le rôle du plaidoyer et la mise en exergue des avantages comparatifs du nouveau paquet de services pour les SAA. En effet, ces interlocuteurs, en relatant les faits majeurs qui ont marqué les péripéties de l'introduction des soins après avortement dans leurs pays respectifs, sont revenus largement sur le rôle de certaines personnes, dont l'engagement aura permis d'amener des acteurs et des décideurs à comprendre et à soutenir le processus qu'ils voulaient mettre en œuvre. Le rôle des RO qui avaient été réalisées au Burkina Faso et au Sénégal a été aussi magnifié, dans la mesure où, selon les responsables interviewés, celles-ci ont fourni des informations clés sur les avantages du modèle de SAA, qui ont facilité la prise de décision, même dans les autres pays.

\footnotetext{
${ }^{9}$ Questions aux soins après avortement. Extension des services en Afrique francophone. Rapport analytique de la conférence régionale francophone sur les SAA. 2004. 82 p.
} 
Il faut, cependant, noter que dix (10) ans après l'introduction des SAA en Afrique francophone, le contexte a changé, puisqu'il est admis que l'approche est maintenant acceptée dans la plupart des pays. De ce fait, il semble qu'il faille, selon le pays, faire l'économie de certaines étapes qui, naguère, paraissaient incontournables ; ou bien, les alléger. D'ailleurs, quelques fois, des initiatives privées ont devancé la mise en place officielle du programme. Ce changement de contexte a révélé l'importance d'autres enjeux qui sont liés à la généralisation et à la pérennisation de l'offre de soins après avortement, à travers le passage à l'échelle et/ou la décentralisation, l'institutionnalisation, le renforcement des capacités des ressources humaines, la supervision, l'approvisionnement en matériel et la continuité de l'offre de services.

\section{La mise à l'échelle et/ou la décentralisation des SAA}

Dans tous les pays visités, la volonté de rendre disponibles les SAA sur toute l'étendue du territoire a été affirmée par tous. Cependant dans certains pays on parle d'amener les SAA jusqu'au sein des communautés, dans d'autres comme le Mali, cela n'est pas encore à l'ordre du jour. Au demeurant, les schémas de décentralisation et ou de passage à l'échelle sont aussi différents, certains pays ayant adopté une approche centralisée au niveau national, supposant un plan et un interlocuteur uniques pour tous les acteurs et partenaires. D'autres, par contre, ont privilégié une approche décentralisée, appelant les collectivités locales à définir et mener leurs propres stratégies. Cette dernière option semble être la plus porteuse, dans la mesure où elle responsabilise d'office les entités décentralisées dans la définition et le financement de leurs stratégies et actions, au contraire de l'approche centralisée, avec laquelle les bailleurs continuent à être sollicités. Ceci, soit pour l'approvisionnement, soit pour la formation des prestataires. Néanmoins, la mise en œuvre de l'approche décentralisée ne serait réussie que si un cadre législatif et/ou juridique clair, autorisant un transfert de ressources et de compétences en termes de gestion administrative et financière, est préalablement établi.

\section{L'institutionnalisation des SAA}

Tous les pays ont élaboré ou mis à jour leur(s) document(s) de Politiques, Normes et Protocoles, incluant les SAA. Dans certains pays, ce document avait inclus les SAA, avant la mise en place du modèle. Suite à la conférence de 2002, les PNP pour les SAA ont été établis sur la base des 5 composantes, ou bien sont en voie de l'être. Il faut toutefois relever une inadéquation entre les PNP et les textes généraux régissant le secteur de la santé, notamment au Sénégal, où le partenariat avec les communautés figure dans les PNP, pour le volet SAA, et des actions sont sensées être menées et supervisées en direction des cases de santé et de leur personnel. Cependant, le système de santé de ce pays ne compte pas les cases de santé communautaires parmi les structures de santé. Ce qui fait, qu'en principe, c'est l'infirmier chef de poste de santé qui est sensé superviser les activités des cases, mais celles-ci ne sont généralement pas comptabilisées dans le système d'information sanitaire. Donc, il faudrait, là où la situation l'exige, mettre en harmonie les textes généraux du système de santé et les PNP, afin de réaliser une institutionnalisation et une décentralisation cohérente.

Dans plusieurs sites il n'existe pas réellement de système de collecte codifié et institutionnalisé ; la qualité de la collecte dépend essentiellement de la volonté d'une ou de quelques personnes. Ceci, bien que partout, les données relatives aux SAA sont systématiquement enregistrées et, le plus souvent, sur des registres standardisés et quelques fois imprimés. Le problème réside, en partie, dans la diversité des points de traitement des complications d'avortement, surtout lors des gardes. Les supports de données pour les SAA n'étant pas disponibles en ces moments là, 
beaucoup d'activités post avortement n'y sont pas consignées. La solution devrait alors consister à mettre à la disposition du personnel de garde le registre des activités de SAA, en veillant, toutefois, sur la qualité du remplissage. Un extrait des informations consignées sur ce support pourrait alors être reporté sur le registre du point de prestation (salle d'accouchement ou bloc opératoire).

\section{Formation des prestataires}

Malgré la reconnaissance du renforcement des compétences des ressources humaines comme étant un des aspects clés des programmes de SAA, on ne peut pas parler de manière formelle, de l'existence d'une politique en la matière, dans un des pays de l'étude. Comme pour toute innovation, afin de répondre au besoin de passage à l'échelle, c'est par le biais de la formation continue que la plupart des agents acquièrent leurs compétences, avec le soutien financier des partenaires. Or la viabilité d'une telle approche n'est pas garantie à cause de la mobilité du personnel compétent, qui entraîne un recommencement permanent. Pour faire face à ce problème, deux approches ont été identifiées. Dans la première, les prestataires déjà formés aident leurs collègues à acquérir les compétences nécessaires pour prendre en charge les complications d'avortement. Néanmoins, si cette approche semble avoir donné des résultats intéressants dans certains pays, elle n'a pas bien fonctionné dans d'autres, comme le Sénégal. Tout de même, de par son potentiel important, relevé par plusieurs acteurs, cette approche mériterait d'être documentée et appuyée, le cas échéant, par la mise en place d'un mécanisme d'encadrement et de validation des gestes effectués par les stagiaires. Cela permettrait de former l'ensemble du personnel de jour et de garde. La seconde approche consiste à introduire les modules de formation en SAA dans les curricula des institutions de formation des médecins, sages-femmes et/ou infirmiers. Les différents pays en sont à des étapes plus ou moins avancées de gestation de leurs projets respectifs. Les institutions partenaires devraient appuyer davantage ce processus par le plaidoyer et l'assistance logistique, surtout pour le développement des capacités des sites de stage.

\section{Supervision}

La difficulté de mettre en place un système de supervision pour les activités de SAA efficace et pérenne a été soulevée par tous les interlocuteurs. Ce sont soit le manque de moyens, soit le manque de ressources humaines compétentes, soit le caractère non exclusif des supervisions, qui en sont à l'origine. En fait, le problème pourrait avoir une origine structurelle, sachant que, d'une part, la supervision des activités cliniques est prévue aussi bien par le système de santé, dans ses activités régulières, que par les programmes qui sont sensés l'appuyer, et est souvent confiée à la même équipe. Or, il arrive très souvent que les objectifs et méthodes des programmes ne cadrent pas avec la disponibilité et les possibilités réelles des équipes de supervision qui font face à de nombreuses sollicitations de programmes divers. Ce trop plein d'activités ne permet pas une supervision approfondie sur un aspect donné. Ce qui est contraire aux exigences d'une bonne supervision formative, tel que le demandent les programmes de SAA. D'ailleurs la notion de supervision formative pose un problème plus ardu en termes de ressources humaines. En effet, dans ce contexte, le superviseur doit avoir un profil de formateur pour pouvoir identifier les défaillances et les corriger. On ne retrouve souvent pas ce profil parmi les membres des équipes de district et de région chargés de superviser les activités de SAA. Il serait donc plus judicieux

que les formateurs s'occupent de la supervision formative, et que les équipes de région et de district procèdent, plus fréquemment, à la supervision facilitatrice qui semble être plus à leur portée. La maîtrise de cette dernière forme de supervision ne nécessiterait qu'une séance 
d'orientation, au lieu d'une longue série de formations coûteuses et dont le résultat pourrait ne pas être garanti à cause de la mobilité du personnel.

\section{Approvisionnement et équipement en matériel de SAA}

Le problème de l'approvisionnement en matériel de SAA, plus précisément les seringues AMIU se pose avec acuité dans les pays visités. En effet, les produits sont généralement fournis par les institutions partenaires dont les programmes ne sont pas pérennes. D'un autre côté, les systèmes de santé nationaux de santé ne sont pas encore parvenus à mettre en place des mécanismes efficaces et durables d'acquisition de matériels de SAA, bien que des solutions transitoires aient été parfois tentées. Actuellement, les solutions à l'étude, ou bien qui sont sur le point d'être mises en œuvre consistent à inscrire la seringue AMIU dans la liste des matériels essentiels. C'est le cas en Guinée.

En attendant, des structures périphériques (centres de santé ou leur équivalent) ont réussi à mettre en place des dispositifs qui leur permettent d'assurer leurs approvisionnements en kits AMIU sur fonds propres, grâce à des systèmes de recouvrement des coûts. Ces exemples réussis devraient inspirer les structures du même type, étant donné que leurs systèmes de gestion sont assez légers, et que les maternités occupent généralement un rôle de premier plan dans la génération des ressources de ces centres.

Pour les hôpitaux, à cause de leurs nombres importants de services et de leur mode de gestion centralisé, il faudrait un effort de sensibilisation et de plaidoyer auprès des administrations respectives, afin de les amener à considérer les SAA comme une composante à part entière de leurs catalogues de services et non plus comme un programme externe.

\section{Organisation des services et prestations offertes}

Bien que les SAA soient dispensés en continu dans les sites visités, les unités de soins après avortement ne sont généralement accessibles qu'aux heures et jours ouvrables. Le reste du temps, les patientes sont prises en charge par les équipes de gardes. A ce propos, des différences apparaissent dans la qualité de l'offre de services, dans la mesure où le personnel de garde n'a souvent ni le temps, ni le matériel nécessaire pour effectuer correctement et complètement la prise en charge des complications d'avortement. Pour régler le problème des ressources humaines, des structures se sont organisées, afin qu'il figure toujours au moins un prestataire formé en SAA dans chaque garde. Par ailleurs, dans plusieurs structures, les locaux des unités de SAA sont fermés en dehors des heures de service normales, et le matériel d'AMIU y est conservé. De ce fait, les équipes de garde n'ont pas accès au matériel de traitement et de counseling, mais aussi aux produits contraceptifs. Ils font alors recours à la dilatation et curetage et au curage digital pour procéder à l'évacuation utérine, sans pouvoir assurer un counseling, ni offrir une méthode contraceptive.

Dans la plupart des sites, la fermeture des unités de SAA s'explique par un souci d'éviter les dérives. En fait, ces unités de SAA sont souvent situées à des endroits assez éloignés des salles d'accouchement ou des blocs opératoires. Elles restent donc souvent isolées par rapport au circuit habituel des personnels de garde. Dans ces conditions, il ne serait pas facile d'en contrôler les entrées et sorties et l'utilisation du matériel. D'un point de vue psychologique, l'"isolement" de l'unité de SAA peut inconsciemment faire croire au personnel que cela continue d'être un programme à part. Une solution serait donc de rapprocher les unités de SAA des blocs de soins obstétricaux et néonataux d'urgence (salle d'accouchement et bloc opératoire), afin 
que l'accès au matériel AMIU, son conditionnement et l'accès au matériel de counseling, aux produits contraceptifs et aux supports de collecte de données puissent être gérés dans le cadre d'une garde normale. Cette solution n'exclut pas la séparation de l'unité de SAA d'avec les autres services étant donné que cela a permis un accès direct des patientes aux soins et leur a ainsi évité d'être mises en concurrence avec d'autres types de complications gynécologiques ou obstétricales qui sont souvent considérées comme prioritaires.

Par ailleurs, les coûts des prestations demeurent élevés, quel que soit le type de structure de traitement, selon les prestataires et aussi pour les patientes. Ceci est paradoxal, dans la mesure où le paquet de services, surtout avec l'AMIU, est sensé rendre plus accessible les SAA au plan financier. Il convient, donc, de revisiter l'ensemble des procédures, de même que le circuit des patientes, afin d'identifier les sources de dépense superflues.

\section{Counseling}

La possibilité pour les équipes de garde de faire le counseling en PF représenterait une avancée notoire dans l'accès continu des femmes à des services de qualité. Il reste toutefois à renforcer les interactions entre les patientes et les prestataires avant et pendant le traitement. Pour ce faire, l'on devrait développer des outils et guides spécifiques destinés aux prestataires, à l'image du counseling pour la PF. En fait, cette innovation introduite avec les SAA, devrait rayonner sur l'ensemble des prestations de service médicales et paramédicales.

\section{Offre de méthodes contraceptives}

Toutes les structures visitées offrent des méthodes contraceptives. Cependant pour les femmes traitées pour une complication d'avortement, dans certaines formations sanitaires la méthode est offerte immédiatement, par contre dans d'autres, les patientes sont référées à l'unité de PF après le counseling. Cette entité peut-être située dans la structure sanitaire ou en dehors. Dans d'autres structures encore (au Sénégal), les produits contraceptifs sont achetés par les patientes au niveau de leur dépôt pharmaceutique. Cette dernière situation s'explique par l'inscription des produits contraceptifs sur la liste des médicaments essentiels. Pourtant, une étude réalisée au Kenya en 1996 et 1997 a montré que l'offre de méthode contraceptive par le personnel traitant, immédiatement après l'évacuation utérine, offre les meilleures garanties d'acceptation et d'utilisation de la contraception ${ }^{10}$. C'est d'ailleurs cette approche qui a été utilisée lors des recherches opérationnelles et qui continue d'être enseignée aux prestataires. Il faudrait donc convaincre les responsables des structures de santé de mettre des méthodes contraceptives dans les unités de SAA, avec un système efficace de recouvrement des coûts. La gamme de méthodes devrait être la plus large possible, à l'image de celle disponible dans les unités de PF.

\section{Liens avec d'autres services}

Le counseling et l'offre de méthode contraception sont un des points forts des SAA. Car, l'un des objectifs majeurs des programmes est de prévenir les grossesses non désirées et les avortements répétitifs par la planification familiale. De ce point de vue, la référence des femmes traitées pour

\footnotetext{
${ }^{10}$ Solo, Julie, et al. 1998. «Détermination de la relation entre le traitement d'une interruption de grossesse incomplète et les services de planification familiale au Kenya : quelle est la meilleure approche? » voir aussi « Amélioration des soins post abortum destinés aux patients des hôpitaux, résumés de RO, janvier 1998 ».
} 
une complication d'avortement vers les unités de PF devrait être systématisée. De plus, il devrait être intéressant de créer des liens étroits entre les SAA et d'autres programmes tels que celui de lutte contre le cancer du col utérin et celui de lutte contre le paludisme.

Pour la lutte contre le cancer du col utérin, il s'agit plutôt de contribuer à la prévention en procédant à la détection systématique des présentations anormales au moment du traitement après avortement, par la méthode d'inspection visuelle à l'acétique ou au lugol. Cela permettrait d'alléger les coûts, aussi bien pour les patientes que pour les structures chargées du diagnostic de confirmation.

Pour la lutte contre le paludisme, il s'agirait de prévention, mais aussi de traitement, car cette maladie représente une des principales causes d'avortement. En pratique la relation avec le programme de lutte contre le paludisme se concrétiserait par un appui au dépistage du parasite, le traitement le cas échéant, et l'offre de moustiquaire imprégnée.

\section{Partenariat avec les communautés}

L'établissement de liens entre les SAA et les programmes de santé intéressant la mère et l'enfant, légitime davantage l'implication des communautés dans l'amélioration de l'accès aux soins pour ces cibles. En effet, en plus du fait que les communautés ont désormais un rôle dans la reconnaissance des signes d'avortement et l'organisation des évacuations, on note, de même, que toutes les activités de santé basées sur la prévention (ou l'incluant) ont, comme interlocutrices principales, les communautés. Toutefois, peu d'actions ont été initiées par les responsables des programmes de SAA en direction des communautés. Jusqu'à présent seules quelques institutions partenaires se sont investies dans ce domaine, pour lequel aucun pays n'a encore développé ou mis en œuvre un plan d'action. La sensibilité de la question ne serait pas étrangère à ce manque d'initiative. Une solution serait donc de mettre en place au niveau communautaire des programmes à volets multiples, en y incluant les SAA. La jonction avec le programme de lutte contre le paludisme pourrait représenter une autre porte d'entrée.

Dix ans après l'introduction des SAA en Afrique francophone, les promoteurs de cette stratégie ont franchi de nombreuses étapes qui font son succès actuel. Cependant, plusieurs défis continuent à se dresser sur le chemin de sa généralisation et de sa pérennisation. A travers cette évaluation, les enjeux les plus importants ont été identifiés. Ils trouvent pour l'essentiel leur origine dans l'engouement de plus en plus grand que le modèle suscite parmi les décideurs et les populations. L'évaluation a aussi permis d'identifier des pistes porteuses de solutions probablement avantageuses. Il convient, dès lors, de pousser la réflexion et de mener les actions idoines, afin d'accompagner et de parachever l'œuvre entreprise. 


\section{REFERENCES BIBLIOGRAPHIQUES}

1. Bulletin médical de l'IPPF. Volume 41. Numéro 3. Septembre 2007. pp 3.

2. Détermination de la relation entre le traitement d'une interruption de grossesse incomplète et les services de planification familiale au Kenya : quelle est la meilleure approche ? Solo, Julie, et al. janvier 1998.

3. Evaluation de l'extension des services de soins après avortement au Sénégal, Population Council, Ministère de la Santé et de la Prévention, Centre de Formation et de Recherche en Santé de la Reproduction (CEFOREP), USAID. 2007. 30 p.

4. Extension des Soins Après Avortement (SAA) : Etude de la référence et de quelques aspects des SAA dans les hôpitaux régionaux de Kaolack, Diourbel et dans le Centre de Santé de Sokone. Ministère de la Santé, Centre de Formation et de Recherche en Santé de la Reproduction (CEFOREP), Fonds des Nations Unies pour la Population (UNFPA) ; 2001 ; $35 \mathrm{p}$.

5. Questions aux soins après avortement. Extension des services en Afrique francophone. Rapport analytique de la conférence régionale francophone sur les SAA. PAC Consortium, USAID. 2004. 82 p.

6. Réunion des points focaux de l'Initiative Francophone Africaine pour les Soins Après Avortement. Cotonou. 2004. 51 p.

7. Réunion du Groupe de Travail pour la Revitalisation des Soins Après Avortement (SAA) : Mise à jour des mécanismes de soutien et suivi pour le renforcement des services SAA en Afrique Francophone. Rapport de synthèse. Dakar. 2006. 


\section{ANNEXES}

\section{Responsables de programmes et d'institutions interviewés}

\section{Burkina Faso :}

- M. Lat Sémé Médina, Directeur des Etudes de l'Institut National de Santé Publique

- Dr Charlemagne Ouédraogo, Médecin Chef de la maternité du CMA du Secteur 30 à Ouagadougou

- Dr Azara Bamba, Conseillère au Bureau de l'Organisation Mondiale de la Santé à Ouagadougou

- Dr SANOU Dieynaba OUEDRAOGO, Direction de la Santé de la Famille à Ouagadougou

- Mme Dieynaba DIALLO, Coordonnatrice de Family Care International au Burkina Faso

- Dr BASSANE Birahima, chargé de projet Initiative aux soins qualifiés à Family Care International, dans le district de Wargadj

- Pr Blandine THIEBA, responsable adjointe de la maternité du CHU Yalgado OUEDRAOGO, membre du CRESAR et point focal SAA du Burkina Faso

- Pr Bibiane KONE, ancienne chef de service de la maternité du CHU Yalgado OUEDRAOGO, Ancienne Présidente du CRESAR du Burkina Faso

- Dr Olga Sankara, chargée de programme au bureau de l'UNFPA à Ouagadougou

\section{Guinée :}

- Dr Bademba Diallo : Responsable de la Formation au Ministère de la Santé de Guinée

- Dr Bouba Touré : Chargé de Programme EngenderHealth

- Dr Gouma : Chef de section à la DSR

- Dr Kouyaté : Médecin Chef du Centre Médical Communal de Konakry

- Pr Yolande Hydjazi : Enseignante à la Faculté de Médecine. Chef de Service Adjoint de Gynécologie à l'Hôpital Donka de Konakry. Point Focal SAA Guinée

\section{Mali :}

- Dr Bineta Keïta : Chef DSR

- Dr Boubacar Traoré : Point focal SAA

- Pr Amadou Dolo : Chef de Service de Gynécologie Hôpital Gabriel Touré

- Pr Mamadou Traoré : Médecin Chef Centre de référence Commune 5

\section{Niger :}

- Mr Ali Boukary : Administrateur Family Care International

- Dr Madi Nayama : Médecin Chef Maternité Issaka Gazoby de Niamey

\section{Togo :}

- Pr Koffi Akpadza : Enseignant Faculté de Médecine. Chef de service de Gynécologie, Hôpital Tokoin de Lomé

- Dr Ntapi Kassouta : Directeur de la Direction de la Santé de la Reproduction 


\section{Prestataires interviewés}

\section{Burkina Faso :}

- Dr Thiémoko OUATTARA, gynécologue au CHU Yalgado OUEDRAOGO de Ouagadougou

- Dr Djibril Kabré - CHU Yalgado à Ouagadougou

- Dr Issaka Kaboré - Gynécologue au CMA du Secteur 30 à Ouagadougou

- Dr Lidjo Sabine - Gynécologue au CMA du Secteur 30 à Ouagadougou

- Sage-femme Responsable unité SAA du CHU Yalgado

- Sage-femme à l'unité SAA

- Sage-femme responsable de l'unité SAA du CMA du Secteur 30 à Ouagadougou

- Sage-femme à l'unité SAA du CMA du Secteur 30 à Ouagadougou

\section{Mali CHU}

- Dr Abdourahmane SAMAKE CHU Gabriel TOURE

- Dr Niani MOUNKORO CHU Gabriel TOURE

- Mme DIANE Hawa Soumaré CHU Gabriel Touré

- Mme TRAORE Fanta Fofana CHU Gabriel Touré

\section{Mali périphérie}

- Dr Oumar TRAORE Centre de Référence Commune 5

- Dr Zoumana TRAORE Centre de Référence Commune 5

- Mme TOURE Kadiata Doumbia Centre de Référence Commune 5

- Mme TOURE Fatoumata MAÏGA Centre de Référence Commune 5

\section{Guinée CHU}

- Dr Hawa CONDE CHU Donka

- Dr Ibrahima SYLLA CHU Donka

- Dr Suzanne AUSTIN CHU Donka

- Mme BALDE Salimatou CHU Donka

\section{Guinée périphérie}

- Dr KABA Mariama GBE Centre de Santé Municipal la Minière

- Dr Nfamara CAMARA Centre de Santé Municipal la Minière

- Mme Christine THEA Centre de Santé Municipal la Minière

- Mme Mariama Bineta DIALLO Centre de Santé Municipal la Minière

\section{Togo}

- Pr Koffi Akpadza, Gynécologue, Chef de service de la maternité de l'hôpital Tokoin de Lomé

- Dr Adama Hondegla

\section{Niger}

- Dr Fatima Zahra Diallo : Médecin Chef Adjoint Maternité Issaka Gazoby de Niamey

- Mme Ganda Balkissa : Sage-femme à l'unité de SAA de la maternité Issaka Gazobi de Niamey

- Mme Nana Aïchatou Sani : Sage-femme à l'unité de SAA de la maternité Issaka Gazobi de Niamey

- Mme Issa Aïssa : Sage-femme à l'unité de SAA de la maternité Poudrière de Niamey

- Mme Kadidja Coulibaly : Sage-femme à l'unité de SAA de la maternité Poudrière de Niamey 